\title{
Stratified Flows over and around Long Dynamically Tall Mountain Ridges
}

\author{
ArJun Jagannathan, Kraig Winters, And Laurence ARmi \\ Scripps Institution of Oceanography, University of California, San Diego, La Jolla, California
}

(Manuscript received 21 May 2018, in final form 12 February 2019)

\begin{abstract}
Uniformly stratified flows approaching long and dynamically tall ridges develop two distinct flow components over disparate time scales. The fluid upstream and below a "blocking level" is stagnant in the limit of an infinite ridge and flows around the sides when the ridge extent is finite. The streamwise half-width of the obstacle at the blocking level arises as a natural inner length scale for the flow, while the excursion time over this half-width is an associated short time scale for the streamwise flow evolution. Over a longer time scale, low-level horizontal flow splitting leads to the establishment of an upstream layerwise potential flow beneath the blocking level. We demonstrate through numerical experiments that for sufficiently long ridges, crest control and streamwise asymmetry are seen on both the short and long time scales. On the short time scale, upstream blocking is established quickly and the flow is well described as a purely infinite-ridge overflow. Over the long time scale associated with flow splitting, low-level flow escapes around the sides, but the overflow continues to be hydraulically controlled and streamwise asymmetric in the neighborhood of the crest. We quantify this late-time overflow by estimating its volumetric transport and then briefly demonstrate how this approach can be extended to predict the overflow across nonuniform ridge shapes.
\end{abstract}

\section{Introduction and background}

The study of stratified flow past topography is of practical interest in atmospheric science and oceanography. Applications include parameterizing surface drag and turbulence in the lee of ridges, forecasting orographic precipitation, and predicting the occurrence of downslope windstorm events. For a recent review of atmospheric applications, see Chow et al. (2012). While flow over dynamically short obstacles is fairly well described in terms of linear theory (e.g., Queney 1948; Smith 1980; Durran 1990), flow over dynamically tall obstacles is fundamentally nonlinear in character. Here dynamically tall implies a mountain height larger than the intrinsic height scale obtained from the upstream flow speed and stratification.

Further, when the cross-stream length of the ridge is large compared to its along-stream width, flows "over" the crest and "around" the sides develop over disparate time scales. Here, we consider long and dynamically tall mountain ridges and quantify the evolution of the overflow as the low-level fluid upstream splits and flows laterally around the sides of the obstacle.

Corresponding author: Arjun Jagannathan, agjagann@ucsd.edu
Energetics arguments (Sheppard 1956) show that, for a sufficiently slow flow or strong stratification, much of the air upstream and below the ridge crest remains blocked. Upstream blocking has been observed both in 2D laboratory towing experiments (Baines 1977; Baines and Hoinka 1985) as well as in atmospheric flow over mountains, for example, in flow over the Alps by Armi and Mayr (2007). The dynamical aspects of these flows are reviewed by Jackson et al. (2013).

When the flow is strictly two-dimensional, blocking is accompanied by the formation of an overlying flowing layer that plunges down the obstacle as a nonlinear, hydraulically controlled downslope flow. Continuity and mass conservation require that the flow within this layer makes up for the volume transport deficit caused by upstream flow stagnation. Winters and Armi (2014) show using nonlinear stratified hydraulic theory that, for an infinite obstacle with upstream blocking, the steady-state optimally controlled flow has an upstream velocity profile that is parabolic in shape and this flowing layer thins and accelerates as it plunges down the lee slope. A schematic of this flow is shown in Fig. 1.

One might ask whether hydraulic dynamics persist when the cross-stream ridge length is finite but large. In this case, the upstream fluid that is blocked when the 


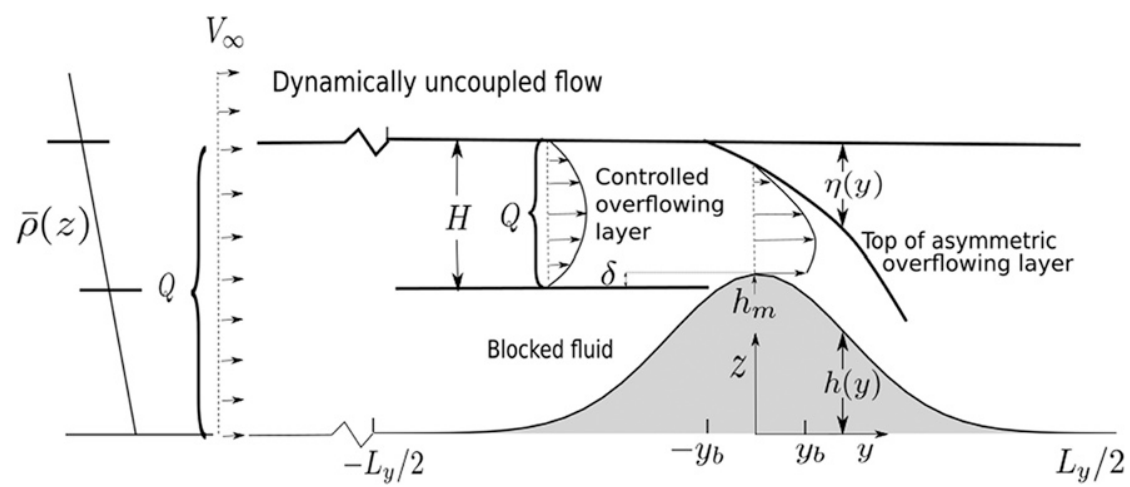

FIG. 1. Schematic of low Fr controlled asymmetric overflow over an infinite ridge. The farupstream flow configuration is characterized by a linear density profile and uniform flow speed. The fluid upstream and below a depth $\delta$ from the crest is blocked. Also $y=-y_{b}$ is the streamwise coordinate of the blocking location, $y=y_{b}$ is the symmetric downstream location, and $Q$ denotes the volume transport within the parabolic overflow, which matches the farupstream transport as shown. The thickness $H$ of the overflow is coupled to its transport $Q$ as $Q=N H^{2} / \pi$ (Winters and Armi 2014). Additionally, on either side of the crest, the vertical plunge $\eta(y)$ of the top of the overflow is uniquely mapped to $h(y)-h\left(-y_{b}\right)$, the height of the topography relative to the blocking point (see also section 3 and appendix A). The streamwise computational boundaries are $y=-L_{y} / 2$ and $y=L_{y} / 2$. At the upstream boundary a radiation condition is prescribed (see section $2 b$ ), which allows upstream-propagating waves to escape the domain without reflection.

ridge is infinite has energetically free horizontal pathways to pass around the obstacle. The validity of a twodimensional treatment of flow past finite obstacles was first raised by Brighton (1978) in the context of laboratory towing experiments. To illustrate the underlying dynamics, we perform numerical experiments in which a uniform upstream flow with speed $V_{\infty}$ and stability $N$ approaches a mountain ridge of height $h_{m}$. We focus on dynamically tall and long ridges, characterized by low topographic Froude number, $\mathrm{Fr}=V_{\infty} / N h_{m} \ll 1$ and large cross-stream to along-stream aspect ratio, $\beta=\sigma_{x} / \sigma_{y} \gg 1$; $\beta$ may also be regarded as a scaled ridge length. Figure 2 shows a schematic of the flow configuration.

Fundamental inner flow scales emerge naturally from this configuration. Energetics considerations (e.g., Sheppard 1956) suggest that the upstream flow remains blocked below a depth $\delta \approx V_{\infty} / N$ from the crest. We refer to $\delta$ as the blocking scale. When $\mathrm{Fr} \ll 1, \delta \ll h_{m}$ constitutes an inner vertical length scale for the overflow. The flow below the blocking level $z=h_{m}-\delta$ is either blocked or splits around the obstacle laterally, suggesting a second inner length scale $\sigma_{y_{\delta}}=\mathscr{O}\left(\operatorname{Fr} \sigma_{y}\right)$, the half-width of the

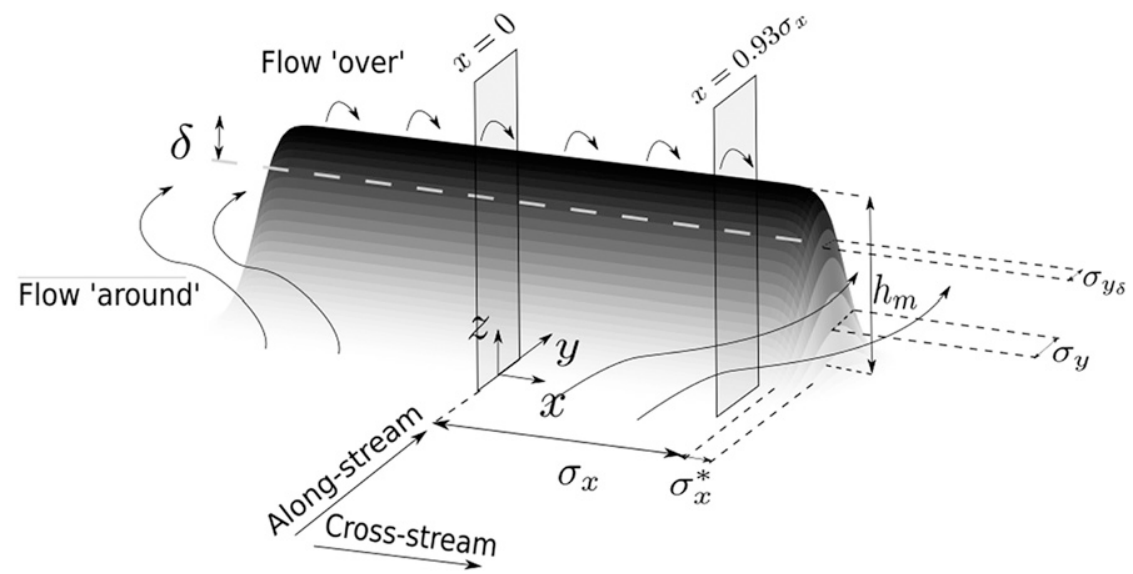

FIG. 2. Flow schematic for low Fr flow past a long but finite ridge of height $h_{m}$, along-stream half-width $\sigma_{y}$, and cross-stream half-length $\sigma_{x}$ with $\beta=\sigma_{x} / \sigma_{y} \gg 1$ that falls steeply to ground level at the lateral ends within a length scale $\sigma_{x}^{*} \ll \sigma_{x}$. The far-upstream flow speed and stratification are uniform and equal $V_{\infty}$ and $N$, respectively, with $\mathrm{Fr}=V_{\infty} / N h_{m} \ll 1$. Figure is not to scale. 
obstacle at the blocking level. The fluid above the blocking level has sufficient kinetic energy to flow over the ridge. Thus $\beta_{\delta}=\sigma_{x} / \sigma_{y_{\delta}}$ is an effective scaled ridge length for the flow component that crests the obstacle. The excursion time,

$$
t_{\delta}=\frac{\sigma_{y_{\delta}}}{V_{\infty}}
$$

then follows as a natural inner time scale. Note that the inner time scale can also be rewritten as $t_{\delta}=\mathscr{O}\left(\operatorname{Fr} \sigma_{y} / V_{\infty}\right)=$ $\mathcal{O}\left(\sigma_{y} / N h_{m}\right)$. This suggests another interpretation of $t_{\delta}$ as the time taken by columnar internal wave modes (cf. Baines 1987) of vertical scale $h_{m}$ and speed $\mathscr{O}\left(N h_{m} / \pi\right)$ to propagate an upstream distance $\sigma_{y}$. These columnar modes promote flow blocking below $z=h_{m}-\delta$ and an accelerated overflow across the crest.

Beneath the blocking level, the upstream flow eventually evolves to a layerwise horizontal potential flow (e.g., Drazin 1961). Thus the half-length $\sigma_{x}$ of the ridge is the appropriate outer length scale. Assuming that flow splitting is also accomplished by columnar internal wave modes of vertical scale $h_{m}$ that communicate the finite extent of the ridge to a distance of about $\sigma_{x}$ upstream,

$$
t_{\beta}=\frac{\sigma_{x}}{N h_{m} / \pi}
$$

is an outer time scale for the low-level splitting flow. A summary of these key dimensional scales and nondimensional parameters is provided in Table 1.

A factor that influences the fate of plunging downslope flows is the presence or absence of a dense, cold pool downstream of the crest. In low Fr flow past finite ridges, the low-level dense fluid in the lee is retained and recirculates slowly. This manifests as a pair of vertically oriented lee vortices that have been observed in numerical simulations (e.g., Smolarkiewicz and Rotunno 1989) and laboratory experiments (Hunt and Snyder 1980). As we will see, the retention of a cold pool inhibits plunging in the lee.

Drazin (1961) developed asymptotic solutions for flows with $\mathrm{Fr} \ll 1$ in which, to leading order in small $\mathrm{Fr}$, the steady state is a layerwise potential flow at all depths below the blocking level. However, this steady asymptotic solution does not give insight into the mechanisms or time scales involved in its establishment, nor does it contain a vortical wake structure. Epifanio and Durran (2001) considered long ridges with $\beta$ up to 12 focusing their attention on the unblocked flow regime, $\mathrm{Fr} \geq 1$ for which $\beta_{\delta}=\beta$. Flows with $\mathrm{Fr}<1$ over obstacles with $\mathscr{Q}(1)$ horizontal aspect ratios have been studied by a number of authors (e.g., Smolarkiewicz and Rotunno 1989; Hunt and Snyder 1980; Hanazaki 1988). In this regime,
TABLE 1. Summary of important dimensional inner and outer

\begin{tabular}{|c|c|c|}
\hline Term & Description & Units \\
\hline \multicolumn{3}{|c|}{ Dimensional outer parameters } \\
\hline$V_{\infty}$ & Basic flow speed & $\mathrm{ms}^{-1}$ \\
\hline$N$ & Background stratification & $\mathrm{s}^{-1}$ \\
\hline$h_{m}$ & Ridge height & $\mathrm{m}$ \\
\hline$\sigma_{y}$ & Streamwise ridge half-width & $\mathrm{m}$ \\
\hline$T=\sigma_{y} / V_{\infty}$ & Outer excursion time scale & $\mathrm{s}$ \\
\hline$\sigma_{x}$ & Cross-stream ridge half-length & $\mathrm{m}$ \\
\hline$\sigma_{x}^{*}$ & $\begin{array}{l}\text { Length of lateral end/connecting } \\
\text { sections }\end{array}$ & $\mathrm{m}$ \\
\hline$t_{\beta}=\sigma_{x} /\left(N h_{m} / \pi\right)$ & Outer flow-splitting time scale & s \\
\hline \multicolumn{3}{|c|}{ Dimensional inner parameters based on the blocking scale } \\
\hline$\delta \approx V_{\infty} / N$ & Blocking scale & $\mathrm{m}$ \\
\hline$h_{m}-\delta$ & Blocking level & $\mathrm{m}$ \\
\hline$\sigma_{y_{\delta}}$ & $\begin{array}{l}\text { Streamwise ridge half-width at } \\
\text { blocking level }\end{array}$ & $\mathrm{m}$ \\
\hline$t_{\delta}=\sigma_{y_{\delta}} / V_{\infty}$ & Inner excursion time scale & s \\
\hline \multicolumn{3}{|c|}{ Dimensionless outer quantities } \\
\hline $\mathrm{Fr}=V_{\infty} / N h_{m} \ll 1$ & Topographic Froude number & - \\
\hline$\beta=\sigma_{x} / \sigma_{y}$ & Scaled ridge length & - \\
\hline$t^{\prime}=t / T$ & Scaled outer excursion time & - \\
\hline$t_{\beta}^{\prime}=t / t_{\beta}$ & Scaled outer splitting time & - \\
\hline \multicolumn{3}{|c|}{ Dimensionless inner quantities } \\
\hline $\mathrm{Fr}_{\delta}=V_{\infty} / N \delta \approx 1$ & Overflow Froude number & - \\
\hline$\beta_{\delta}=\sigma_{x} / \sigma_{y_{\delta}}$ & $\begin{array}{l}\text { Scaled dynamic ridge length for the } \\
\text { overflow }\end{array}$ & - \\
\hline$t_{\delta}^{\prime}=t / t_{\delta}$ & Scaled inner time & - \\
\hline
\end{tabular}
variables and dimensionless flow parameters.

splitting flows are established quickly; consequently 2D solutions are not very useful in characterizing them. Previous investigations of low Fr flow past elongated ridges have been confined to moderate values of $\beta \leq 5$ (Bauer et al. 2000; Ólafsson and Bougeault 1996). The linear regime diagram of Smith (1989) provides some guidance as to the $\mathrm{Fr}$ and $\beta$ ranges over which one might expect "wave breaking" and "flow splitting" but as pointed out by Smith (1989) and others (Bauer et al. 2000; Ólafsson and Bougeault 1996) there are uncertainties associated with the regime boundaries for low Fr and large $\beta$.

To investigate the extent to which flow features characteristic of the infinite-ridge solution such as crest control and streamwise asymmetry are seen in low Fr flows past long but finite ridges, we performed a suite of numerical experiments. We first consider a $\mathrm{Fr}=0.16$ flow over an infinite ridge and demonstrate that, by prescribing a uniform outflow with a radiation condition upstream, the flow rapidly evolves toward the optimally controlled downslope flow of Winters and Armi (2014). We then consider the same flow over a finite ridge with steep ends for which $\beta=30$. We show that the streamwise flow near the ridge center is well described as an infinite-ridge overflow for a finite time after which it starts to diverge owing to splitting effects. We quantify 
the evolution by measuring the volume transport in the overflow as a function of time. Over longer $\mathscr{Q}\left(t_{\beta}\right)$ time scales, we show that, by reformulating the overflow transport to account for lateral flow splitting, the late-time flow over the ridge can still be described by stratified hydraulic theory. Finally, considering an example of a ridge with an abrupt change in height, we demonstrate how these general principles can be extended to predict the overflow across nonuniform ridges.

\section{Modeling approach}

We consider three-dimensional, nonrotating, incompressible flow, with free-slip boundary conditions on the ridge surface. The numerical experiments are performed using the spectral large-eddy solver described in Winters and de la Fuente (2012), with the bottom topography incorporated as a smooth immersed boundary. The goal is to capture the essentially inviscid dynamics at the large scales of the flow. To this end, we employ a sixth-order hyperdiffusion operator to explicitly diffuse only the motions near the smallest grid scale. At rest, the fluid is in hydrostatic balance everywhere, with

$$
\frac{\partial \bar{p}_{0}(z)}{\partial z}=-\bar{\rho}_{0}(z) g
$$

where $\bar{p}_{0}(z)$ is the static pressure, $\bar{\rho}_{0}(z)$ is the initial density profile, and $g$ is the acceleration due to gravity. The fluid is stably stratified with uniform buoyancy frequency $N$ and a density difference $\Delta \rho$ across the total fluid depth. Perturbing about this rest state, for the flow in the domain of interest, the nonlinear equations of motion in the Boussinesq limit are

$$
\begin{aligned}
\frac{\partial \mathbf{u}}{\partial t}+\mathbf{u} \cdot \nabla \mathbf{u}+g \frac{\rho^{\prime}}{\rho_{0}} \hat{\mathbf{k}} & =-\frac{1}{\rho_{0}} \nabla p^{\prime}+\nu^{*} \mathscr{D} \mathbf{u}, \\
\frac{\partial \rho}{\partial t}+\mathbf{u} \cdot \nabla \rho & =\kappa^{*} \mathscr{D} \rho, \\
\nabla \cdot \mathbf{u} & =0 .
\end{aligned}
$$

Here, $\mathbf{u}$ is the three-dimensional velocity vector and $\hat{\mathbf{k}}$ is the unit vector in the $z$ direction; $\rho_{0}$ is a constant reference density; $\rho^{\prime}$ and $p^{\prime}$ are the perturbation density and pressure, respectively; and $\rho$ is the total density:

$$
\rho=\rho^{\prime}+\bar{\rho}_{0}(z) .
$$

The topography slopes gently in the streamwise direction with $h_{m} / \sigma_{y}=1 / 6$, rendering the upstream pressure approximately hydrostatic. Note however that we do not invoke the hydrostatic approximation and are thus able to capture $\mathscr{O}(1)$ aspect-ratio shear-induced overturning motions downstream.

In (4b) $\mathscr{D}$ is a sixth-order hyperdiffusion operator,

$$
\mathscr{D} \equiv\left(\frac{\partial^{6}}{\partial x^{6}}+\frac{\partial^{6}}{\partial y^{6}}+\frac{\partial^{6}}{\partial z^{6}}\right),
$$

and $\nu^{*}$ and $\kappa^{*}$ are hyperviscosity and hyperdiffusivity, respectively, which are chosen such that gridscale motions decay to $\exp (-1)$ their value within a dissipation time scale $T_{\text {diss }}=5 \Delta t$, where $\Delta t$ is the model time step.

\section{a. Experimental setup}

In all our experiments, the vertical height $L_{z}$ of the domain is taken to be 6 times the maximum obstacle height. The flow is rapidly accelerated from rest, with the inflow conditions being ramped up to their desired values over $10 \Delta t$.

\section{1) INFINITE-RIDGE EXPERIMENT}

We consider a flow with topographic Froude number $\mathrm{Fr}=0.16$ incident on an infinite Gaussian ridge $(\beta=\infty)$ centered at $y=0$,

$$
h=h_{m} \exp \left(-\frac{y^{2}}{\sigma_{y}^{2}}\right) .
$$

Given Fr, the half-width at blocking depth, $\sigma_{y_{\delta}}$ is found by substituting $h=h_{m}-\delta \approx h_{m}-V_{\infty} / N=h_{m}(1-\mathrm{Fr})$ in the LHS of (7), giving for $\mathrm{Fr}=0.16$,

$$
\sigma_{y_{\delta}} \approx \sigma_{y} / 2.5 .
$$

We set $\sigma_{y}=6 h_{m}$ and the domain width $L_{y}=16.7 \sigma_{y}$, with grid spacing $\Delta y=0.038 \sigma_{y_{\delta}}$ and $\Delta z=0.1 \delta$. With respect to the inner time scale $t_{\delta}$, we declare that the flow has reached quasi-steady state when its bulk properties, namely overflow transport, thickness, and peak speed at the blocking location deviate by less than $1 \%$ over $5 t_{\delta}$. The flow reached quasi-steady state by $t=31.3 t_{\delta}$, and the run was terminated at $t=47.6 t_{\delta}$.

\section{2) FINITE-RIDGE EXPERIMENT}

For the finite-ridge configuration (see Fig. 2), Fr is again set to 0.16 and the ridge height is specified as

$$
h=\left\{h_{1}+0.5 \Delta h\left[1+\tanh \frac{\left(\sigma_{x}-|x|\right)}{\sigma_{x}^{*}}\right]\right\} \exp \left(-\frac{y^{2}}{\sigma_{y}^{2}}\right),
$$

where $h_{1}=0, \Delta h=h_{m}$, and $\beta=\sigma_{x} / \sigma_{y}=30$. The ends are steep, with $\sigma_{x}^{*}=\sigma_{x} / 120$. We exploit the symmetry of 
TABLE 2. Numerical implementation details for each of the flow experiments.

\begin{tabular}{|c|c|c|c|}
\hline & Infinite ridge $(\beta=\infty)$ & Finite ridge $(\beta=30)$ & Two-level ridge \\
\hline \multicolumn{4}{|l|}{ Ridge configuration } \\
\hline$\sigma_{y}$ & $6 h_{m}$ & $6 h_{m}$ & $6\left(h_{1}+\Delta h\right)$ \\
\hline$\sigma_{x}$ & - & $30 \sigma_{y}$ & $30 \sigma_{y}$ \\
\hline$\sigma_{x}^{*}$ & - & $0.25 \sigma_{y}$ & $0.25 \sigma_{y}$ \\
\hline \multicolumn{4}{|l|}{ Computational domain } \\
\hline$L_{y}$ & $16.7 \sigma_{y}$ & $140 \sigma_{y}$ & $140 \sigma_{y}$ \\
\hline$L_{x} / 2$ & - & $4.7 \sigma_{x}$ & $4.7 \sigma_{x}$ \\
\hline$L_{z}$ & $6 h_{m}$ & $6 h_{m}$ & $6\left(h_{1}+\Delta h\right)$ \\
\hline \multicolumn{4}{|l|}{ Grid configuration } \\
\hline$\Delta y$ & $0.038 \sigma_{y_{\delta}}$ & $0.125 \sigma_{y_{\delta}}$ & $0.23 \sigma_{y_{\delta}}$ \\
\hline$\Delta x$ & - & $0.018 \sigma_{x}$ & $0.009 \sigma_{x}$ \\
\hline$\Delta z$ & $0.1 \delta$ & $0.1 \delta$ & $0.2 \delta$ \\
\hline Inflow/outflow sponge-layer thickness & $52 \Delta y$ & $135 \Delta y$ & $74 \Delta y$ \\
\hline Upper-sponge-layer thickness & $0.3 L_{z}$ & $0.3 L_{z}$ & $0.3 L_{z}$ \\
\hline \multicolumn{4}{|l|}{ Time step } \\
\hline$\Delta t$ & $1.6 \times 10^{-4} t_{\delta}$ & $8.9 \times 10^{-4} t_{\delta}$ & $1.1 \times 10^{-3} t_{\delta}$ \\
\hline
\end{tabular}

the flow configuration to compute the flow on the half domain $x \geq 0$ only, with $x=0$ treated as a symmetry boundary. To capture lateral flow splitting, we perform the experiment in a large horizontal domain with inflow and outflow channel lengths set to values slightly bigger than $2 \sigma_{x}$ and the lateral half-length, $L_{x} / 2$ set to $4.7 \sigma_{x}$. The boundary condition at the lateral boundary $x=4.7 \sigma_{x}$ is approximated as no normal flow, and $u=0$ along with zero normal gradients on streamwise velocity, pressure, and density.

The grid resolution is $\Delta y=0.125 \sigma_{y_{\delta}}, \Delta x=0.018 \sigma_{x}$, and $\Delta z=0.1 \delta$ and the run was terminated at $t=48.9 t_{\delta}$. A summary of the computational details for each experiment is given in Table 2 .

\section{b. Near-boundary forcing}

A sponge layer of thickness $L_{z} / 3$ is imposed at the upper boundary through a Rayleigh damping term that absorbs upward radiating waves. Inflow and outflow boundary conditions are implemented as sponging terms that relax to the specified target values over $\mathscr{O}(10-100) \Delta y$. The outflow boundary condition is uniform withdrawal at speed $V_{\infty}$.

Low Fr flows over topography excite columnar internal waves that propagate upstream and alter the oncoming flow. So specifying a uniform inflow condition at the upstream boundary will cause wave reflections that may contaminate the solution in the domain interior, particularly if the inflow channel is not very long. For this reason we apply a radiation condition upstream that maintains the depth integrated transport while allowing the vertical profile of the inflow to evolve in time as upstream propagating waves escape the domain. This is implemented through an iterative scheme that measures the upstream influence at an intermediate location between the ridge center $y=0$ and inflow boundary $y=-L_{y} / 2$. This information is then utilized in a dynamically evolving boundary condition that is imposed via relaxation,

$$
v_{-L_{y} / 2}^{i+1}(x, z)=\alpha v_{-L_{y} / 2}^{i}(x, z)+(1-\alpha) v_{y *}^{i}(x, z),
$$

and similarly for $u$ and $\rho$. Here, $i$ is the time step, $y^{*}$ is the intermediate position $\left(-L_{y} / 2<y^{*}<0\right)$ where the flow is measured, and $\alpha$ is a weighting parameter.

In the finite-ridge case, flow-splitting effects extend a distance of $\mathscr{O}\left(\sigma_{x}\right)$ upstream from the obstacle; accordingly $y^{*}$ is conservatively placed further upstream of the crest at $x=-2.5 \sigma_{x}\left(75 \sigma_{y}\right)$. For low Fr flow over an infinite ridge, upstream influence carried by columnar modes propagates arbitrarily far upstream without any change in amplitude (e.g., Pierrehumbert and Wyman 1985). The measurement location in this case is kept a distance of about $10 \sigma_{y}$ upstream of the crest. In general, the measurement location must be placed appropriately far from the topography to ensure that spurious information from nonpropagating, horizontally decaying wave modes is not relayed to the upstream boundary.

The physical basis for the iterative boundary scheme is that, in these subcritical flows, the energy of the upstream propagating signals is primarily contained in columnar internal wave modes (cf. Baines 1987) excited at the crest, having vertical scale $h_{m}$ and group velocity $\mathscr{Q}\left(N h_{m} / \pi\right)$. The parameter $\alpha$ is therefore chosen as follows. We determine the number of time steps $n^{*}$ it takes 
for a columnar mode with group velocity $4 N h_{m} / \pi$ wave to travel from $y=y^{*}$ to the upstream boundary. We then specify that, with respect to any reference time step $i$, the imposed boundary value $v_{-L_{y} / 2}^{i+n^{*}}(x, z)$ at step $i+n^{*}$ derive $90 \%$ of its information from the measured profile at the previous steps. That is, we set

$$
1-\alpha^{n^{*}}=0.9
$$

By design, this scheme converges to a steady, streamwiseuniform inflow profile that is dynamically consistent with the upstream influence of the topographically excited columnar modes. We found this scheme to be robust for different choices of $\alpha$. For example, changing the RHS of (11) from 0.9 to 0.8 produced a negligible change in the bulk properties of the flow. The advantage of this scheme is that it allows for a much shorter inflow channel than would be possible with a more traditional approach of imposing a uniform inflow along with a wave-damping layer to absorb upstream disturbances. We have performed a test run for the infinite-ridge case using a uniform inflow, a very long inflow channel and wave damping near the boundary and verified that the results in the vicinity of the ridge match the ones obtained using the iterative scheme.

\section{A brief overview of the Winters and Armi (2014) analysis}

Winters and Armi (2014) developed semianalytical solutions for hydraulically controlled flow over an infinite ridge. The solution begins by considering a uniform stratification $N$ and jetlike upstream flow profiles of specified thickness $H$ overlying a stagnant blocked layer, as shown in Fig. 1. Note that this approach is different from the hydraulic treatment of Smith (1985) in that it includes the effect of upstream blocking. Asymmetry is triggered by imposing a streamline bifurcation upstream at the top of the jetlike flow. The downslope flow below the lower branch of the bifurcating streamline is then calculated through integrals of Bernoulli's equation. For a chosen upstream wind profile, the bottommost streamline, which represents the terrain surface, is thus determined by the dynamics rather than being imposed a priori.

Among different jetlike upstream flow configurations considered, it was found that a parabolically sheared velocity profile with a velocity maximum $(3 / 2) N H / \pi$ and associated volume transport $N H^{2} / \pi$ was optimal in the sense that it maximized the blocking scale $\delta$ while minimizing the kinetic energy of the flow.

Note that, although the overflow thickness $H$ is assumed a priori in constructing the optimal solution, $H$ and the transport $Q$ of the overflow are in fact coupled through the control relationship,

$$
Q=N H^{2} / \pi
$$

As we will see below, predicting $H$ from (12) requires an estimation of the overflow transport $Q$. Winters and Armi (2014) also found that the blocking scale is dynamically related to the overflow thickness as $\delta=H / 8$.

An important property of the solution is that, on either side of the crest, it generates a unique one-to-one mapping between the height by which the bifurcating streamline has dropped and the terrain height relative to the blocking level. It is thus a valid solution for arbitrary terrain shapes (appendix A). As in all hydraulic models, the solution of Bernoulli's equation relies on the hydrostatic approximation and thus the precise shape of the bifurcating streamline is unimportant as long as its slope is small. Upstream of the blocking location, the terrain shape is arbitrary and is not part of the solution.

Finally, internal hydraulic jumps are not included in this model and so the bifurcating streamline is perpetually plunging over an apparently "bottomless" terrain. However for real ridges, the downslope flow must return to a subcritical state at some location downstream of the crest. The Winters and Armi (2014) solutions are formally valid until this location.

\section{Diagnostics of the overflow}

Volume transport conservation (see also Fig. 1) requires that, at the blocking location, the transport within the overflow between $z=h_{m}-\delta$ and $z=h_{m}-\delta+H$ match the far-upstream transport below the bifurcating streamline. That is,

$$
N H^{2} / \pi=V_{\infty}\left(h_{m}-\delta+H\right) .
$$

Substituting $\delta=H / 8$ in (13) yields a quadratic for $H$, with coefficients given in terms of the outer dimensional parameters $h_{m}, N$, and $V_{\infty}$,

$$
N H^{2} / \pi=V_{\infty}\left(h_{m}+7 H / 8\right) .
$$

Note that $H$ obtained by solving the quadratic equation, (14), is the analytical prediction of the overflow thickness in the infinite-ridge limit. When the ridge is finite, the fluid below $z=h_{m}-\delta$ can escape around the sides and the overflow transport and thickness shrink accordingly. To quantitatively compare this overflow with the infinite-ridge prediction, we define the volume transport per unit length in a layer of height $H$ starting at the blocking level $z=h_{m}-\delta$ and measured at the upstream blocking location $y=-y_{b}$ (indicated in Fig. 1), 


$$
Q^{\prime}(x, t)=\int_{h_{m}-\delta}^{h_{m}-\delta+H} v\left(x,-y_{b}, z, t\right) d z .
$$

For an infinite ridge, the overflow transport is independent of $x$ and $Q^{\prime}(x, t)$ reduces to $Q^{\prime}(t)$. In this case we expect upstream blocking to cause an early surge in the overflow transport, with $Q^{\prime}(t)$ quickly approaching $Q$. For a long but finite ridge, we anticipate that $Q^{\prime}(x, t)$ will approach $Q$ at early times before it starts decreasing as transport is lost to the low-level lateral splitting flow.

As a quantitative measure of asymmetry, we compare the maximum speed at the downstream location $y=y_{b}$ (indicated in Fig. 1) to the reference speed $V_{\infty}$. An additional measure of asymmetry is the plunging depth $p_{d}(x, t)$, defined as the depth from the ridge crest to which the streamline originating at the upstream blocking level $z=h_{m}-\delta$, plunges down the lee slope before it separates.

\section{Results}

We will evaluate the temporal evolution of the diagnostics developed in section 4. For a long ridge $(\beta \gg 1)$, the inner excursion time scale $t_{\delta}$ is much shorter than the outer flow-splitting time scale $t_{\beta}$. Accordingly, we define a scaled inner time

$$
t_{\delta}^{\prime}=t / t_{\delta}
$$

to quantify the near-crest evolution of the overflow and a scaled outer splitting time

$$
t_{\beta}^{\prime}=t / t_{\beta}
$$

to quantify the development of the low-level horizontal splitting flow.

Upstream blocking, streamwise across-crest asymmetry, overturned isopycnals, and downslope flow acceleration will be visible in planar vertical sections. We will also show images of horizontal sections, which will reveal the establishment of low-level flow splitting.

\section{a. Infinite ridge $(\beta=\infty)$}

We first present results for flow over an infinite ridge with $\beta_{\delta}=\beta=\infty$ and $\mathrm{Fr}=0.16$. The blocking scale for this flow is $\delta \approx V_{\infty} / N=0.16 h_{m}$. Thus most of the air upstream and below the ridge crest is blocked.

Figures $3 \mathrm{a}$ and $3 \mathrm{~b}$ show that with respect to the inner time scale, the upstream flow rapidly evolves to that predicted by $2 \mathrm{D}$ theory. By $t_{\delta}^{\prime}=2$, the velocity profile at the blocking location already begins to approach the analytically predicted parabolic profile of Winters and Armi (2014). By $t_{\delta}^{\prime}=11.7$, the overflow has evolved further toward a parabolic shape and both its peak speed (a)

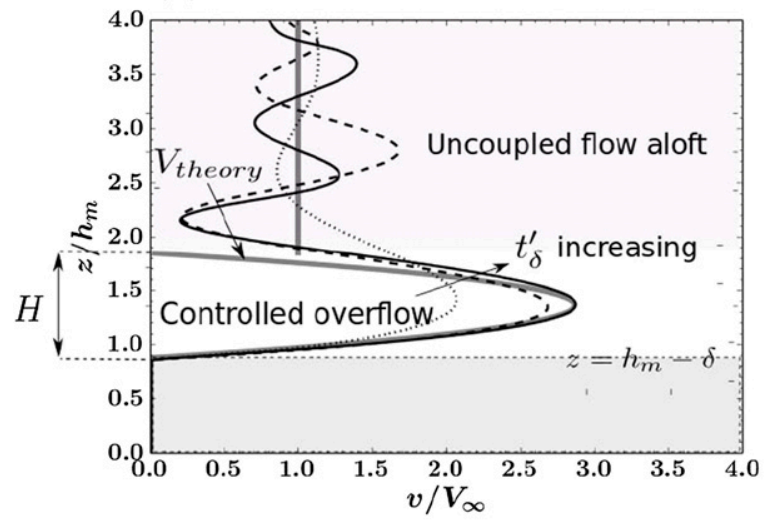

(b)

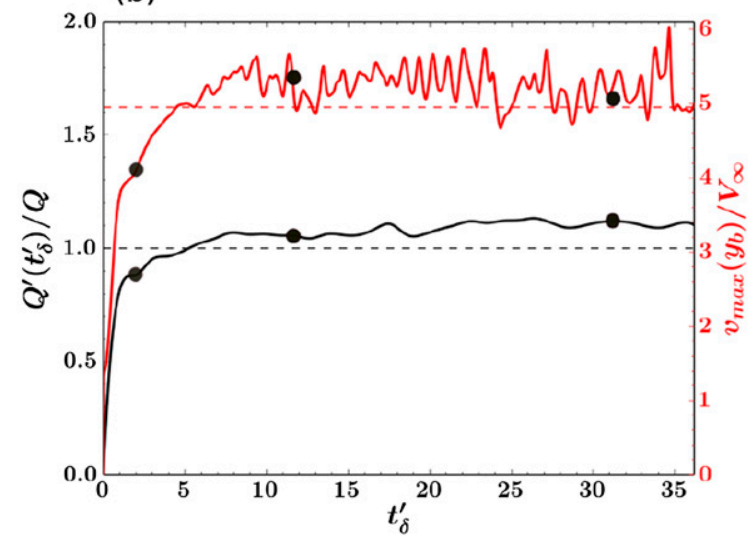

FIG. 3. (a) Vertical profiles of the streamwise velocity at the blocking point $y \approx-y_{b}$ at $t_{\delta}^{\prime}=2.0,11.7$, and 31.1 along with the analytical prediction of Winters and Armi (2014) (gray line) for $\mathrm{Fr}=0.16$ flow over an infinite ridge. The region marked $H$ is the Winters and Armi (2014) prediction of the overflow thickness. (b) Upstream volume transport $Q^{\prime}\left(t_{\delta}^{\prime}\right)$ (black) in the controlled overflowing layer normalized by the theoretical value $Q$ and normalized maximum streamwise velocity, $v_{\max }\left(y_{b}, z, t_{\delta}^{\prime}\right) / V_{\infty}$ (red) at the downstream location $y_{b}$, vs dimensionless time $t_{\delta}^{\prime}$. Dots mark the times at which velocity profiles are shown in (a) and dashed lines are the analytical predictions of Winters and Armi (2014). The location of $y_{b}$ relative to the ridge crest is indicated in Fig. 1.

and volume transport are within $10 \%$ of the analytically predicted values. Later, at $t_{\delta}^{\prime}=31.1$, the peak speed matches the prediction exactly. We remark that the dynamical prediction for the blocking scale $\delta$ obtained from solving for $H$ in (12) yielded a value that is about $25 \%$ smaller than the initial scaling estimate $V_{\infty} / N$.

The time history of $v_{\max }\left(y_{b}\right) / V_{\infty}$ is also shown in Fig. 3b (in red) along with the Winters and Armi (2014) prediction. These infinite ridge solutions are highly asymmetric as indicated by sustained downslope flow speeds of about $5 V_{\infty}$ with gusts approaching $6 V_{\infty}$. These gusts are quasi periodic with a period of about $t_{\delta}$, which for this specific ridge configuration and $\mathrm{Fr}$ is roughly two 
(a)
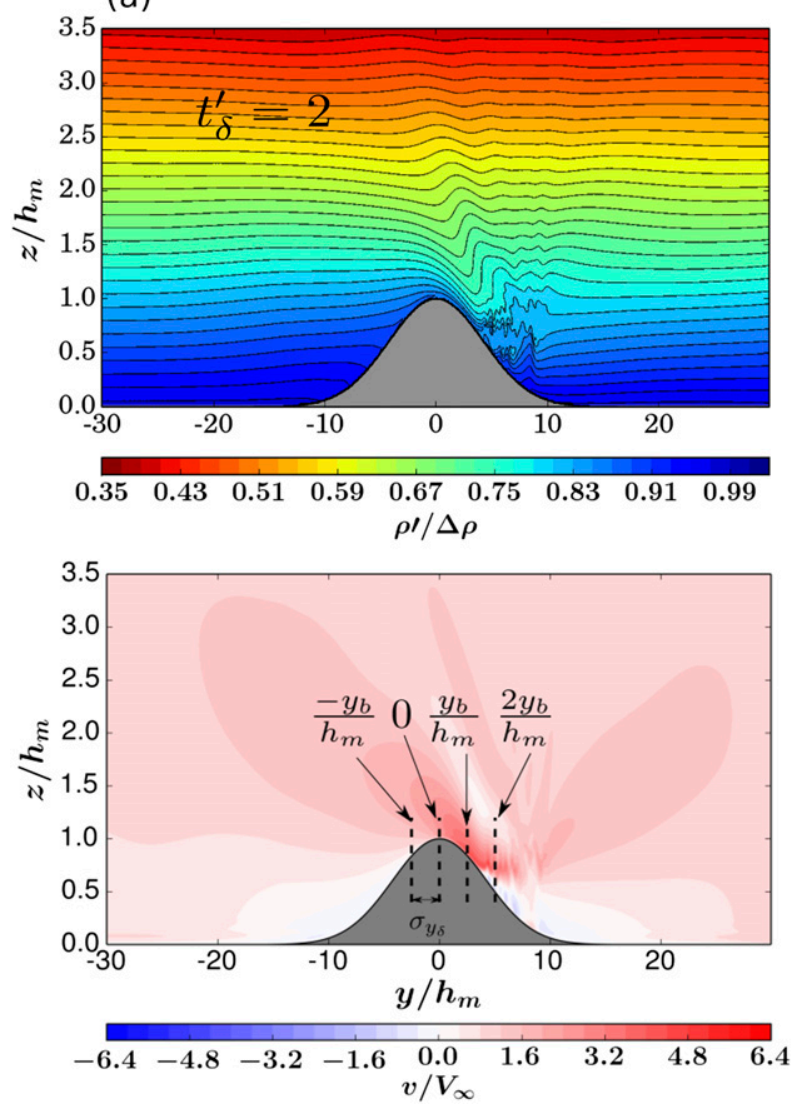

FIG. 4a. Time evolution of the flow field for $\mathrm{Fr}=0.16$ flow over an infinite ridge. Each panel shows (top) isopycnal lines and contours and (bottom) streamwise velocity contours. Also shown in the bottom panel are the position of the blocking location, ridge center, and downstream locations at which we will later (in Fig. 5) display the vertical profiles of the streamwise velocity. Flow is from left to right at $t_{\delta}^{\prime}=2.0$.

buoyancy periods. They are reminiscent of the quasiperiodic gusts observed in the Bora by Belušić et al. (2004) and arise due to Kelvin-Helmholtz (K-H) instability, caused by increasing shear downstream at the top of the overflowing layer (Peltier and Scinocca 1990; Jagannathan et al. 2017).

Figures $4 \mathrm{a}-\mathrm{c}$ show the flow evolution as it approaches a quasi-steady state. Blocking is already visible by $t_{\delta}^{\prime} \approx 2$ and the upstream extent of the blocked flow increases with time. By $t_{\delta}^{\prime}=11.7$, upstream influence in the form of long internal gravity waves has permanently modified the incoming flow, shaping the flow above the blocking level into a parabolic jet. Overturning isopycnals are seen above the crest and further downstream, a plunging downslope flow develops. Both the maximum downslope flow speed and its penetration depth increase with time. The instantaneous snapshot in Fig. 4b reveals turbulent overturns, both aloft and due to K-H instability at the top (b)
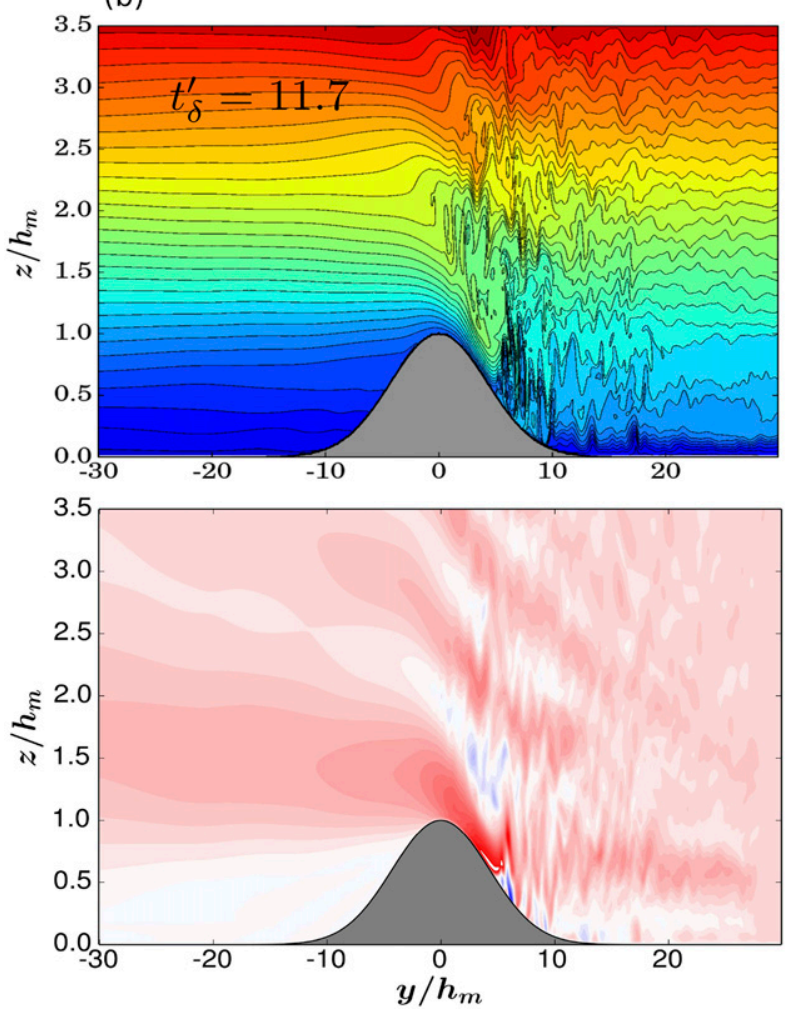

FIG. 4b. As in (a), but at $t_{\delta}^{\prime}=11.7$.

of the unstable overflowing layer (Peltier and Scinocca 1990; Jagannathan et al. 2017). The numerical model, which removes gridscale variability via the hyperdiffusion operator $\mathscr{D}$ does not completely resolve the details of the turbulent mixing due to these processes. Nevertheless, as shown in Fig. 4c, a statistical time average of the quasi-steady flow reveals the essential downstream flow features, which are an accelerating downslope flow and a nearly stagnant, nearly homogeneous isolating layer that separates the downslope flow from the flow aloft. Comparison with Fig. 4b shows that the statistical averaging has no discernible effect on the stable upstream flow.

In their theory, Winters and Armi (2014) do not prescribe a structure for the flow above the overflowing layer, which they assume to be dynamically uncoupled with a mean speed $V_{\infty}$. We note that the computed solutions in Fig. 4 as well as the vertical profiles of $v\left(-y_{b}, z, t_{\delta}\right)$ in Fig. 3a show weak spatial oscillations about this mean which merge smoothly with the jetlike overflow. We have checked that the quantitative features of the controlled overflowing layer as well as the characteristic wavelength $\left(\approx 2 \pi V_{\infty} / N\right)$ of the oscillations aloft are insensitive to the height of the model domain and the thickness of the sponge layer (appendix B). 
(c)
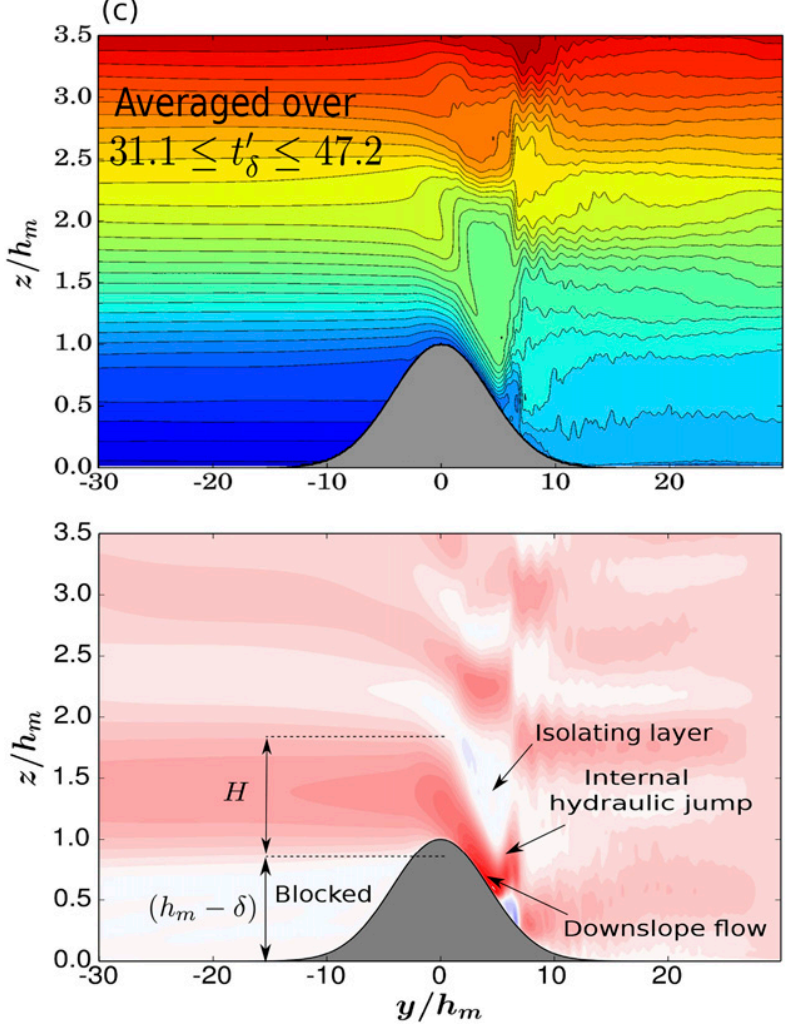

FIG. 4c. As in (a), but averaged over $31.1 \leq t_{\delta}^{\prime} \leq 47.2$.

Across-crest asymmetry sets in early (Fig. 4a) and the stratification becomes increasingly asymmetric across the crest as the flow evolves. By $t_{\delta}^{\prime}=11.7$ (Fig. 4b), the dense air downstream and below the blocking level $z=\left(h_{m}-\delta\right)$ has been almost completely swept away and is replaced by lighter air that has overflowed the crest. This asymmetry in the density field is a consequence of upstream flow blocking and is directly related to the establishment of hydraulic control at the crest. The supercritical flow downstream manifests as an intensifying downslope windstorm.

Depth profiles of the streamwise velocity at and downstream of the crest elucidate the characteristics of the downslope flow. These are displayed at an early and late inner time in Fig. 5. The maximum speed $v_{\max }(y)$ increases downstream and with time at each location as the flow evolves toward a quasi-steady state. At later times, for example, $t_{\delta}^{\prime} \approx 31.9$, the overflow thins and accelerates downstream of the crest. It also progressively plunges deeper in the lee. For example, $v_{\max }\left(2 y_{b}\right) \approx 6.3 V_{\infty}$ and the vertical location of the maximum is at $z \approx 0.6 h_{m}$ which is a depth of about $2.5 \delta$ below crest level. This shows that the flow is highly asymmetric across the crest.

The evolution of the plunging depth $p_{d}\left(t_{\delta}^{\prime}\right)$ of the lowest streamline cresting the obstacle is traced in Fig. 6.

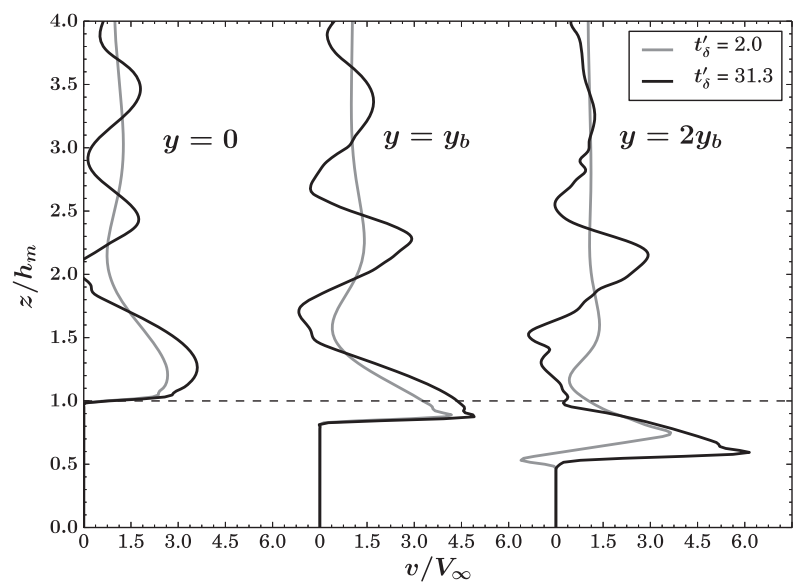

FIG. 5. Vertical profiles of streamwise velocity $v\left(y, z, t_{\delta}^{\prime}\right)$ at various positions downstream of the upstream blocking location $-y_{b}$ for the infinite-ridge overflow at $\mathrm{Fr}=0.16$. The positions of the downstream locations relative to the ridge center are indicated in the bottom panel of Fig. 4a.

The plunging depth reaches sustained values of about $3 \delta$ by $t_{\delta}^{\prime} \approx 4$ and subsequently fluctuates between $2.5 \delta$ and $3.9 \delta$. The fluctuations are associated with the internal hydraulic jump downstream of the separation location.

In summary, low Fr flow over infinite ridges is highly asymmetric across the crest, with respect to both streamwise velocity and stratification. It is characterized by upstream flow blocking and the development of a thinning accelerating downslope flow that plunges down the lee slope to a significant fraction of the ridge height. Within the overflowing layer, the properties of the quasi-steady flow are well described by the stratified hydraulic theory of Winters and Armi (2014).

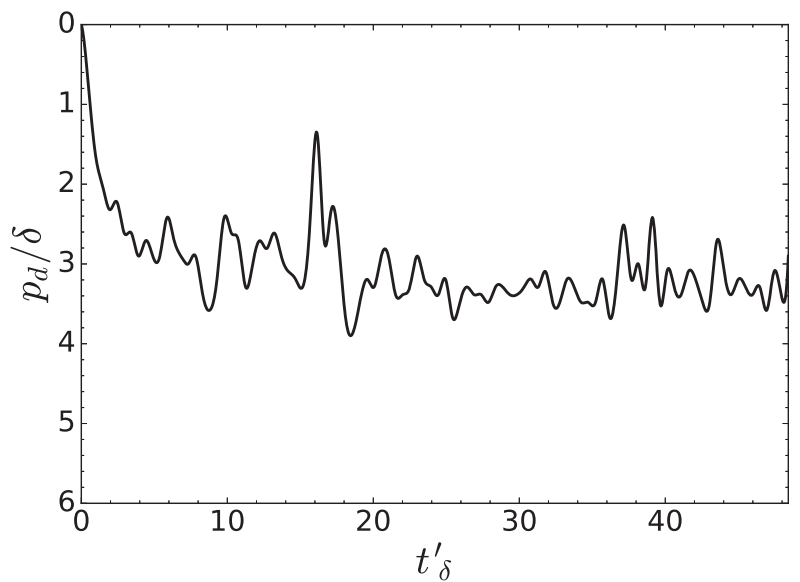

FIG. 6. Plunging depth $p_{d}\left(t_{\delta}^{\prime}\right)$ of the lowest streamline that crests the obstacle for $\mathrm{Fr}=0.16$ flow over an infinite ridge 


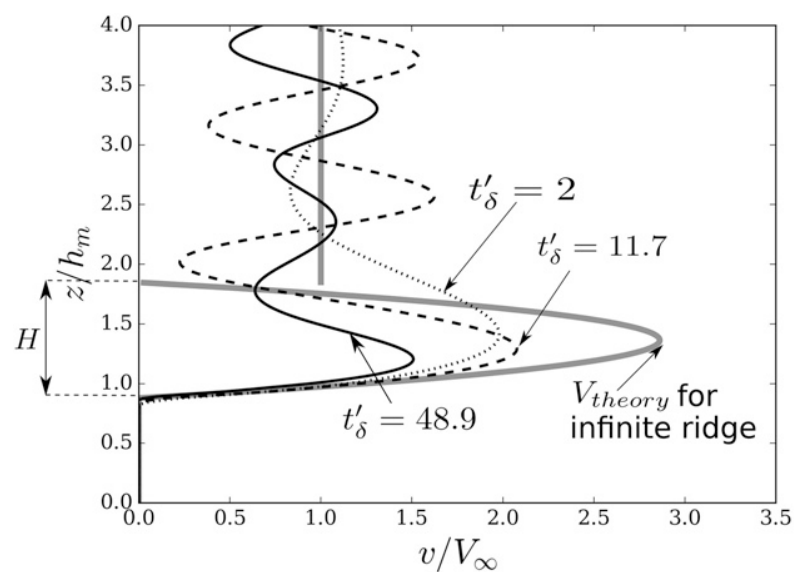

FIG. 7. Vertical profiles of the streamwise velocity $v\left(z, t_{\delta}^{\prime}\right)$ in the center plane, $x=0$, at the blocking point, $y=-y_{b}$, for $\mathrm{Fr}=0.16$ flow over a finite ridge with $\beta=30$. The corresponding Winters and Armi (2014) prediction for the infinite-ridge case at the same Fr is also shown (gray), with $H$ being the predicted overflow thickness.

\section{b. Finite ridge $(\beta=30)$}

We now consider a flow with identical forcing and Froude number incident on a long, but finite ridge with $\beta=30$. The finite extent allows for both flow over and around the ridge and we proceed to quantify how the flow characteristics differ from the infinite ridge case for which a purely $2 \mathrm{D}$ controlled overflow develops. The shape of the ridge is described by (9) with $h_{1}=0$, $\Delta h=h_{m}$, and $\sigma_{x}^{*}=\sigma_{y} / 4$. (see also Fig. 2). We examine the evolution of the overflow in the center plane and at a plane closer to the lateral ends (shown in Fig. 2).

The upstream flow, which crests the obstacle, originates at or above the blocking level $h_{m}-\delta$. Hence the appropriate streamwise length scale for flow over the crest is $\sigma_{y_{\delta}}$, the ridge half-width at blocking level. The scaled dynamic ridge length for the overflow $\beta_{\delta}=\sigma_{x} / \sigma_{y_{\delta}} \approx 75$, which is larger than the geometric-scaled length $\beta=30$ for flow around the sides.

Further, while the topographic Froude number Fr $=$ 0.16 , the appropriate Froude number for the overflow is $\mathrm{Fr}_{\delta}=V_{\infty} / N \delta \approx 1$. Our case is dynamically distinct from $\mathrm{Fr} \approx 1$ flows for which $\beta_{\delta}=\beta$ and $\mathrm{Fr}_{\delta}=\operatorname{Fr}$ (e.g., Epifanio and Durran 2001).

Figure 7 traces the evolution of the streamwise velocity at the upstream blocking location, $v\left(-y_{b}, z, t_{\delta}^{\prime}\right)$ in the symmetric center plane $x=0$. At early times (e.g., $\left.t_{\delta}^{\prime}=2\right)$, the flow profile is nearly identical to that in the infinite-ridge case (Fig. 3a). By $t_{\delta}^{\prime}=11.7$, the profile is similar in structure to the infinite-ridge overflow, but with a lower peak speed. Much later (e.g., $t_{\delta}^{\prime}=48.9$ ), the overflow is considerably thinner and slower than that in the infinite-ridge case. This is because much of the transport associated with the early-time overflow is lost (a)
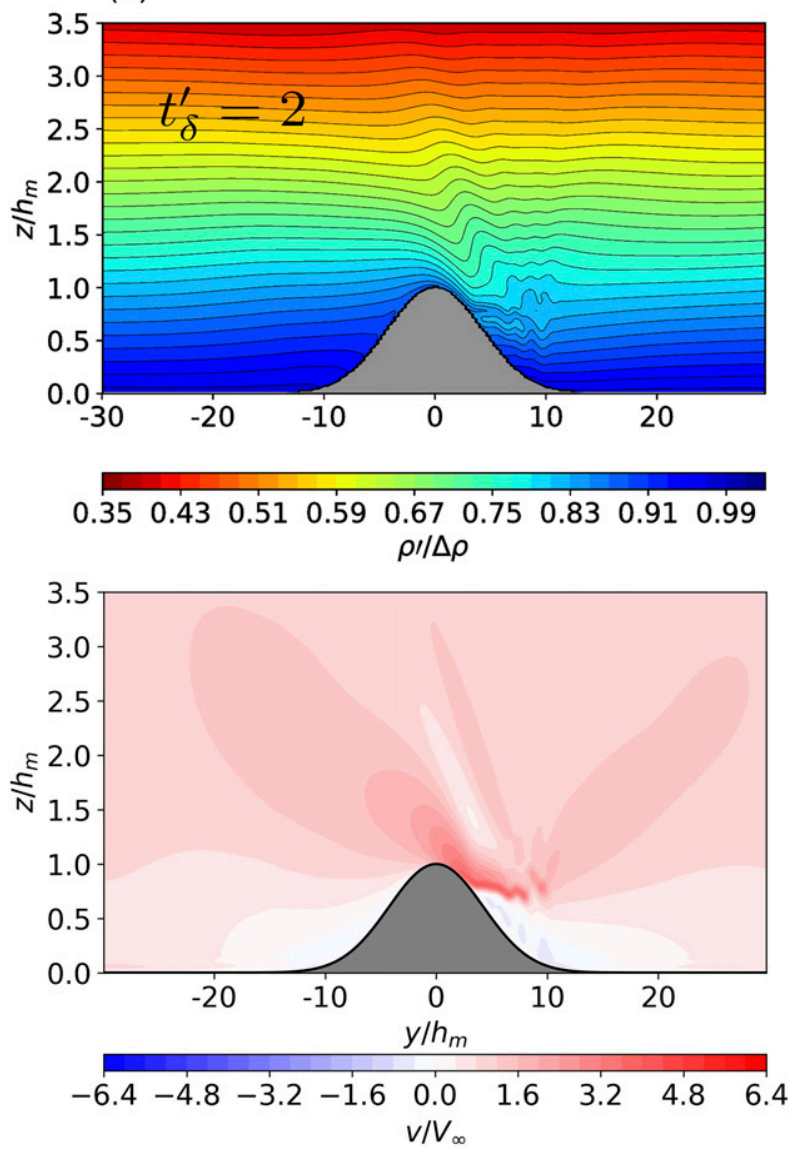

FIG. 8a. Time evolution of the flow field at the symmetric center plane, $x=0$, for $\mathrm{Fr}=0.16$ flow over a finite ridge with $\beta=30$. Each panel shows (top) isopycnal lines and contours and (bottom) streamwise velocity contours. Flow is from left to right at $t_{\delta}^{\prime}=2.0$.

to the low-level horizontal splitting flow. We will show that by a simple reduction of the upstream volume transport in (13), the long-time flow over the ridge can be qualitatively and quantitatively described as an asymmetric crest-controlled overflow.

Figures 8 and 9 show the evolution of the flow field at and away from the ridge center plane, respectively. The stratification and streamwise velocity exhibit asymmetry across the crest in the form of an intensifying downslope flow. Isopycnals overturn above the crest and the flow accelerates as it plunges down the lee slope. At early $\left(t_{\delta}^{\prime}=2\right)$ and intermediate $\left(t_{\delta}^{\prime}=11.7\right)$ times the flow field in the center plane is strongly reminiscent of the infiniteridge solution.

At later times, for example, $t_{\delta}^{\prime}=48.9\left(t_{\beta}^{\prime}=1.3\right)$, the fluid in the lee beneath the blocking level is nearly motionless, both at and away from the center plane (Figs. 8c and 9c). Downslope plunging is strongly inhibited and the stratification below the overflow is nearly symmetric across 
(b)
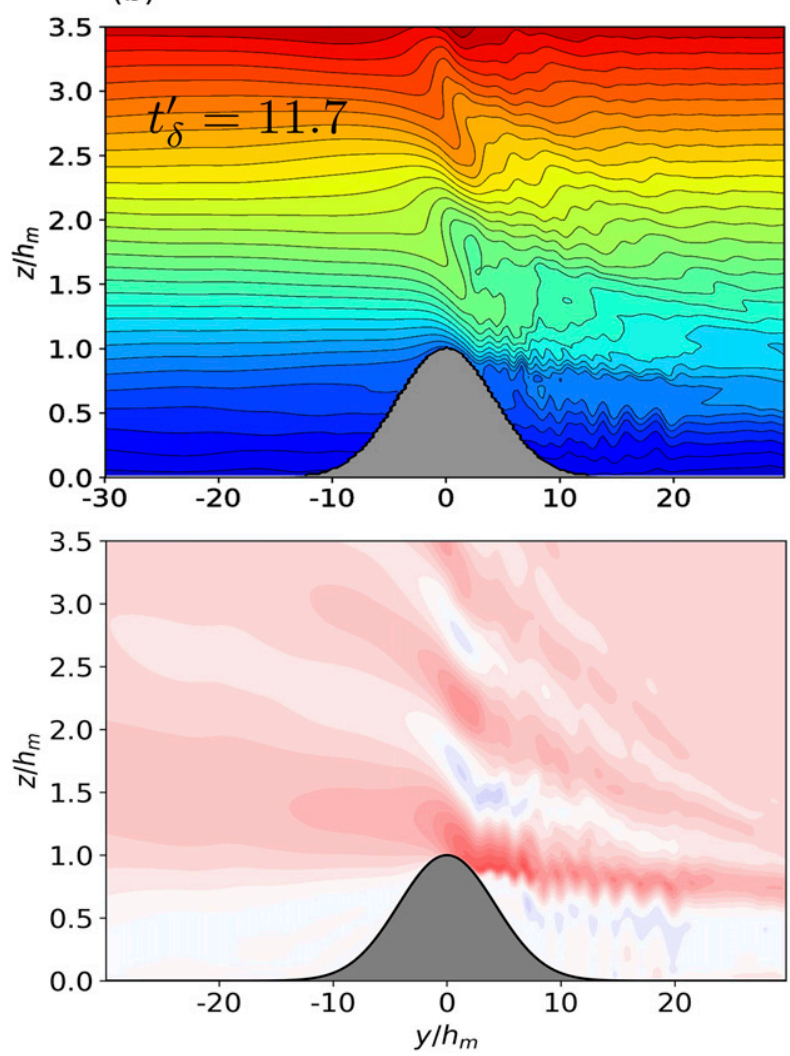

FIG. 8 b. As in (a), but at $t_{\delta}^{\prime}=11.7$.

the crest. The initial dense "cold pool" in the lee is retained on the long flow-splitting time scale. This contrasts strikingly with the infinite-ridge solution (Fig. 4) where the density field becomes highly asymmetric across the crest as the downslope flow evolves. Above the blocking level, the flow is streamwise asymmetric, both at and away from the center plane. This is a clear signature of hydraulic control at the crest. Recall, in addition, that the overflow Froude number $\mathrm{Fr}_{\delta} \approx 1$.

The evolution of horizontal streamlines at $z=0.1 h_{m}$ (i.e., well below the blocking level) is depicted in Figs. $10 \mathrm{a}-$ c. At early outer times (e.g., $t_{\beta}^{\prime}=0.05$ ), the low-level flow is blocked immediately upstream of the ridge. Subsequently, the upstream flow splits laterally around the sides, with speeds of about $2 V_{\infty}$ at the obstacle ends and approaching $3 V_{\infty}$ farther downstream. In the lee, a vortex pair develops and the vortex centers move downstream with time, forming a slowly recirculating flow within the cold pool.

At low levels upstream, the late-time horizontal splitting flow is a layerwise potential flow, as proposed by Drazin (1961). For example, at $z=0.1 h_{m}$, where the ridge half-width is $1.5 \sigma_{y}$, Fig. 11 shows that $v(x)$ at $y=-1.5 \sigma_{y}$ is well approximated by the expression (c)
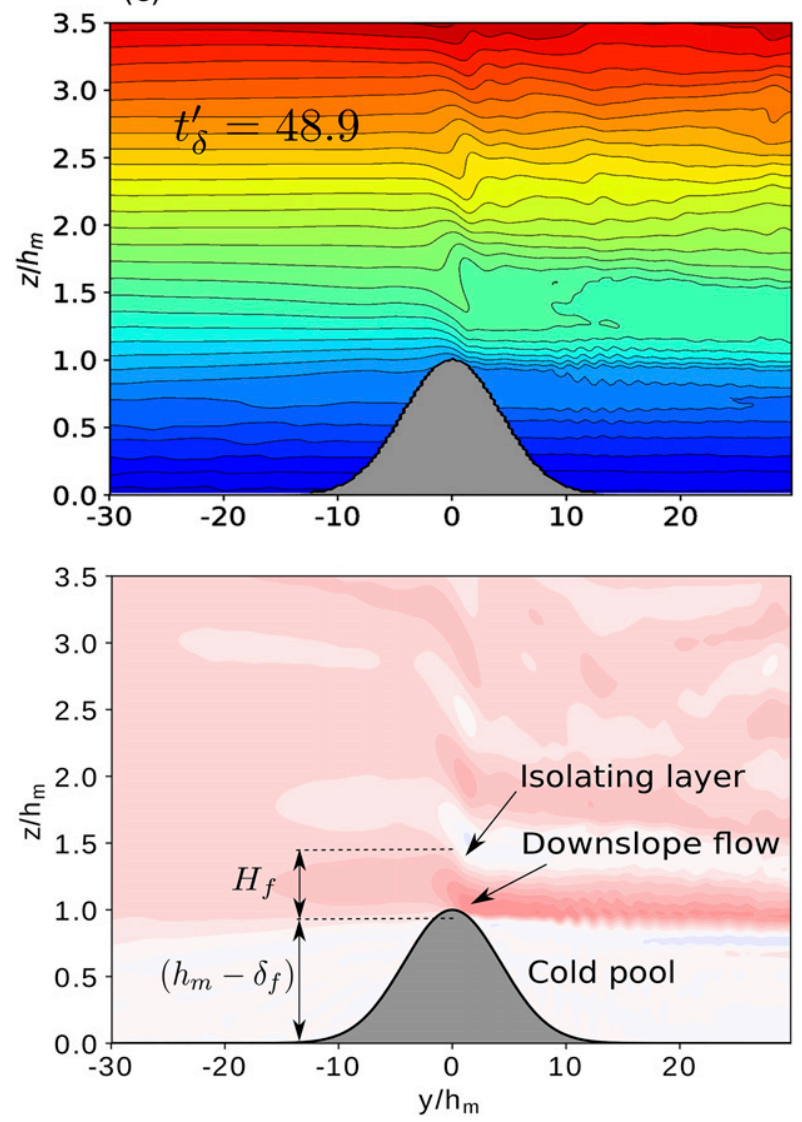

FIG. 8c. As in (a), but at $t_{\delta}^{\prime}=48.9$. The predicted late-time overflow thickness $H_{f}$ and blocking scale $\delta_{f}$ are predicted from (22) and (23), respectively.

$$
v\left(x, y=-1.5 \sigma_{y}\right)=V_{\infty}\left(A+B \frac{\sigma_{x}^{2}}{x^{2}}\right), \quad|x| \geq \sigma_{x} .
$$

This is simply the scaling law for potential flow around convex, symmetric 2D obstacles. The constant $A$ is unity for an infinite domain but is slightly larger here due to the presence of sidewalls, and $B$ is a factor that depends on the details of the obstacle shape. For this case, the best fit was obtained with $A=1.1$ and $B=1$.

As a consequence of low-level flow splitting, the transport in the overflow is less than the infinite-ridge prediction. In Fig. 12, we track the upstream transport, $Q^{\prime}\left(x=0, t_{\delta}^{\prime}\right)$ in a layer of thickness $H$ above the blocking level $h_{m}-\delta$ and compare peak flow speeds at $y_{b}$ with those for the 2D infinite-ridge case. The value of $Q^{\prime}$ deviates from the infinite-ridge curve at $t_{\delta}^{\prime} \approx 1$ by which time it is $85 \%$ of $Q$. From around $t_{\delta}^{\prime}=5, Q^{\prime}$ starts decreasing steadily as more and more transport is lost to the low-level splitting flow. Significantly, at $t_{\delta}^{\prime}=12, Q^{\prime}$ and $v_{\max }\left(y_{b}\right)$ are close to $75 \%$ and $80 \%$ of the predicted 
(a)

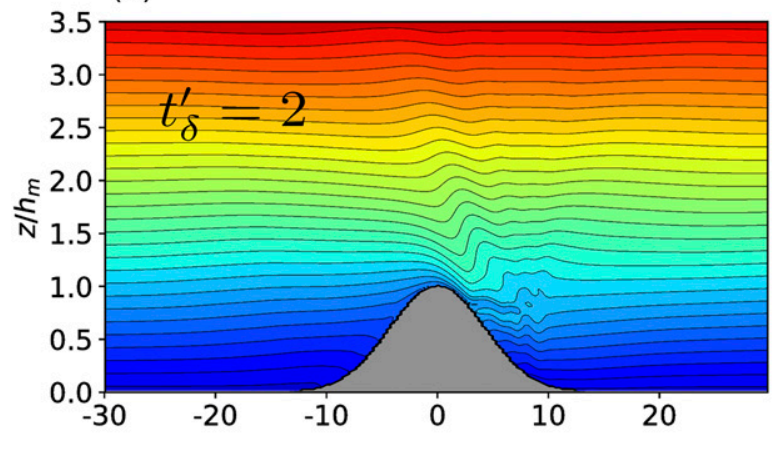

$\begin{array}{lllllllll}0.35 & 0.43 & 0.51 & 0.59 & 0.67 & 0.75 & 0.83 & 0.91 & 0.99\end{array}$ $\rho / / \Delta \rho$

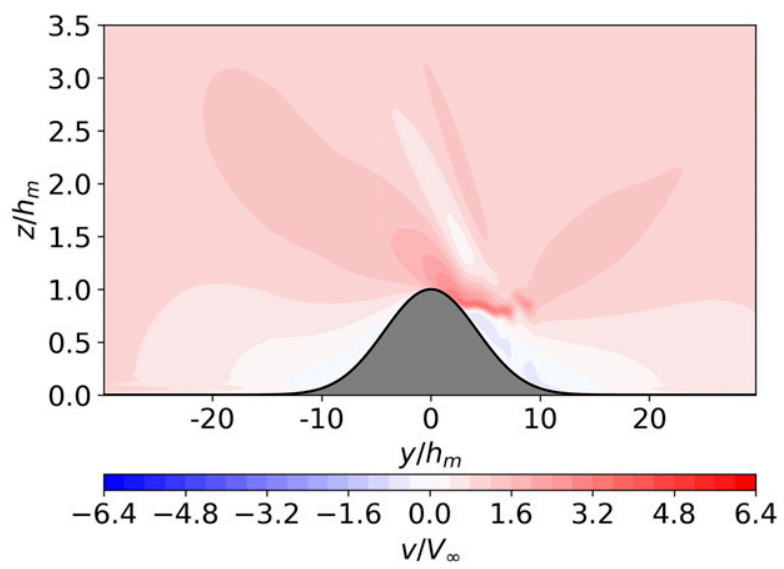

FIG. 9a. As in Fig. 8a, but at the cross-stream location, $x=0.93 \sigma_{x}$.

infinite-ridge values. Therefore for $0<t_{\delta}^{\prime} \leq \mathscr{Q}(10)$, the flow in the center plane is well described as a controlled infinite-ridge overflow.

One distinctive feature of low Fr flows in a purely 2D setting is gustiness of the downslope flow, associated with loss of stability downstream (Jagannathan et al. 2017). This manifests itself as high-frequency oscillations in the measured peak downstream speed $v_{\max }\left(y_{b}\right)$ (Fig. 3b). By contrast, such gustiness is absent in the finite-ridge case. Thus the combination of diminished downslope plunging (Fig. 8) and loss of overflow transport weakens the downstream shear, thereby stabilizing the flow.

At $t_{\beta}^{\prime}=1.3\left(t_{\delta}^{\prime}=48.9\right)$, the flow over the crest is still asymmetric both at and away from the center plane (Figs. $8 \mathrm{c}$ and 9c). By this time, the low-level flow upstream of the ridge has almost entirely split horizontally around the sides of the obstacle (Fig. 10c). Thus at each cross-stream section along the ridge, a portion $V_{\infty}\left(h_{m}-\delta\right)$ of the upstream transport which was absorbed into the overflow at early times is lost to the lateral splitting flow. This motivates the reformulation of the late-time (b)
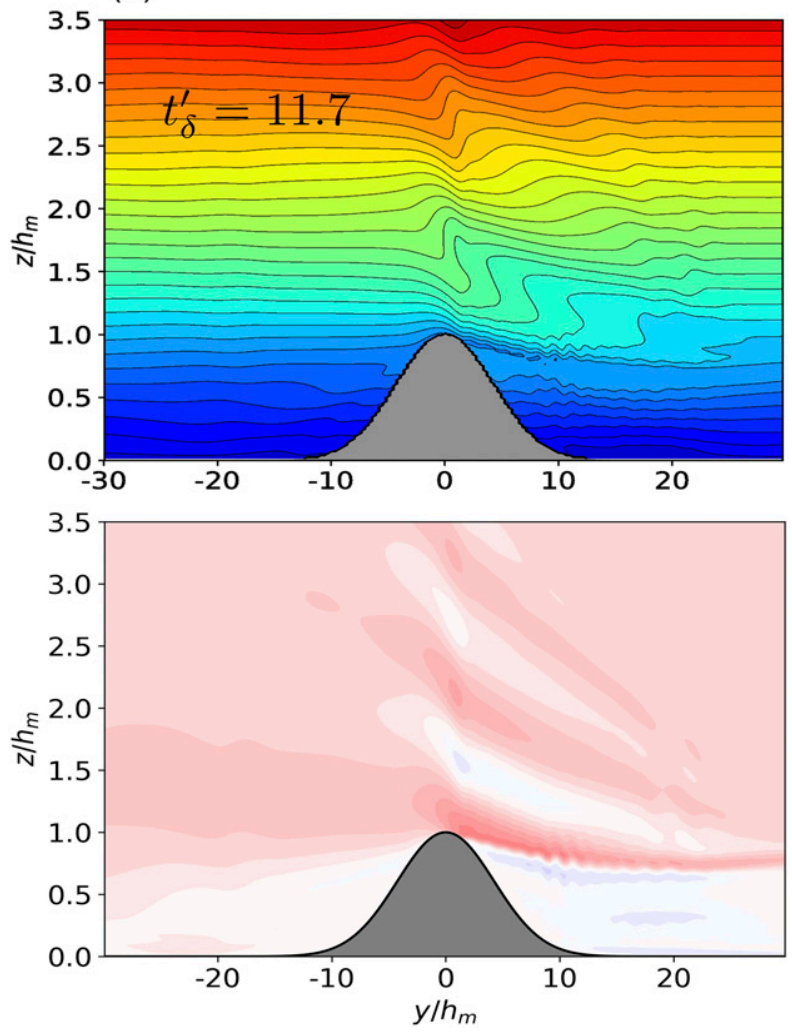

FIG. 9b. As in Fig. 8b, but at the cross-stream location, $x=0.93 \sigma_{x}$.

overflow (Fig. 7) with a reduced transport. From its observed across-crest asymmetry, we infer hydraulic control at the crest. Assuming a modified and as yet unknown thickness $H_{f}$ for this overflow, for optimal control (Winters and Armi 2014), it must be parabolic in shape, with average speed $N H_{f} / \pi$ and transport

$$
Q_{H_{f}}=N H_{f}^{2} / \pi \text {. }
$$

This yields a new blocking scale, $\delta_{f}=H_{f} / 8$. Below $z=h_{m}-\delta_{f}$, the upstream flow is predominantly around the ridge. Recall that the length of the end sections $\sigma_{x}^{*} \ll \sigma_{x}$. Therefore from (18), on either side of the center plane, the excess transport per unit length of the obstacle that escapes around the sides can be written as

$$
Q^{*} \approx \frac{1}{\sigma_{x}} \int_{\sigma_{x}}^{\infty} B V_{\infty} \frac{\sigma_{x}^{2}}{x^{2}}\left(h_{m}-\delta_{f}\right) d x=V_{\infty}\left(h_{m}-\delta_{f}\right),
$$

for the best-fit value $B=1$. This is exactly the amount of transport that is blocked ahead of the ridge at early times. For $\beta_{\delta} \gg 1$, this allows us to estimate the transport lost along each streamwise plane in the ridge interior as $V_{\infty}\left(h_{m}-\delta_{f}\right)$. Therefore the late-time transport for the overflow in the ridge interior is simply $V_{\infty} H_{f}$. 
(c)
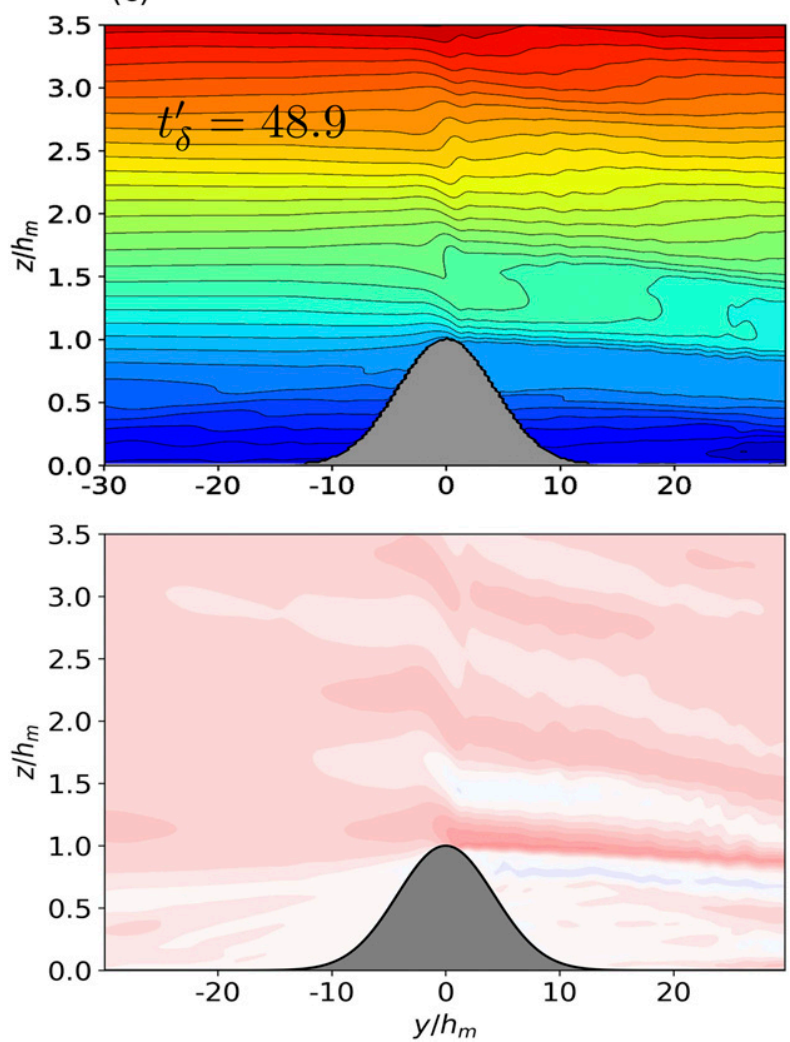

FIG. 9c. As in Fig. 8c, but at the cross-stream location, $x=0.93 \sigma_{x}$

The volume conservation equation, (13), then reduces to

$$
N H_{f}^{2} / \pi=V_{\infty} H_{f}
$$

giving

$$
H_{f}=\pi V_{\infty} / N
$$

and

$$
\delta_{f}=H_{f} / 8=\frac{\pi V_{\infty}}{8 N}
$$

which is more than 2.5 times smaller than the scaling estimate $V_{\infty} / N$, suggesting that the blocking location moves closer to the crest. These predictions for the quantities $H_{f}$ and $\delta_{f}$ match the observed latetime overflow reasonably well, as indicated in Fig. 8c. We denote the new upstream blocking location as $y=-y_{b_{f}}$.

When Fr is small as is the case here, most of the upstream fluid below crest level eventually splits around the ridge. This occurs on the slow time scale $t_{\beta}$ (Fig. 10). Thus, $H_{f}$ is considerably smaller than $H$, which in turn
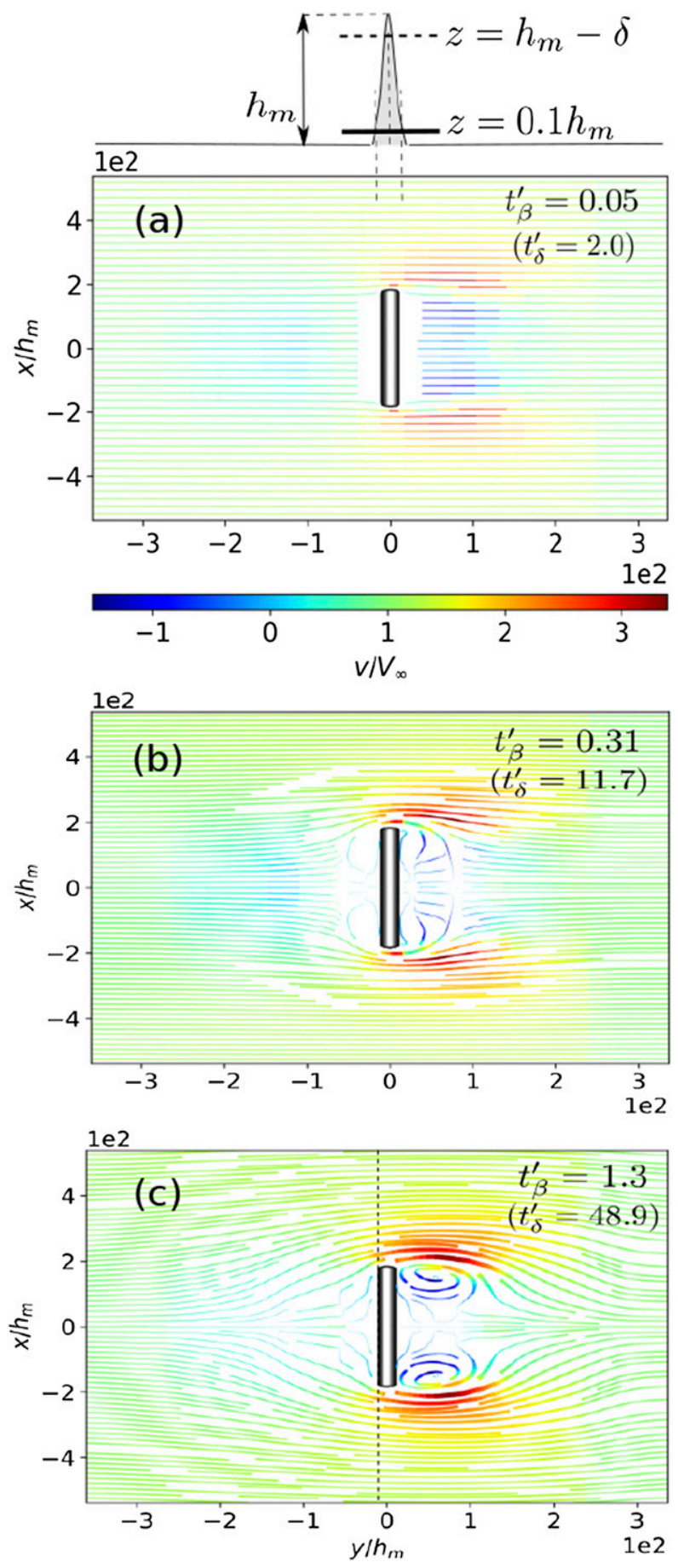

FIG. 10. Time evolution of streamlines in the horizontal plane at $z=0.1 h_{m}$, that is, just above the ground level, for the finite ridge with $\beta=30$ at $\mathrm{Fr}=0.16$ for (a) $t_{\beta}^{\prime}=0.05$, (b) $t_{\beta}^{\prime}=0.31$, and (c) $t_{\beta}^{\prime}=1.3$. Thick red lines indicate fast positive flow, and dark blue lines indicate reversed flow. 


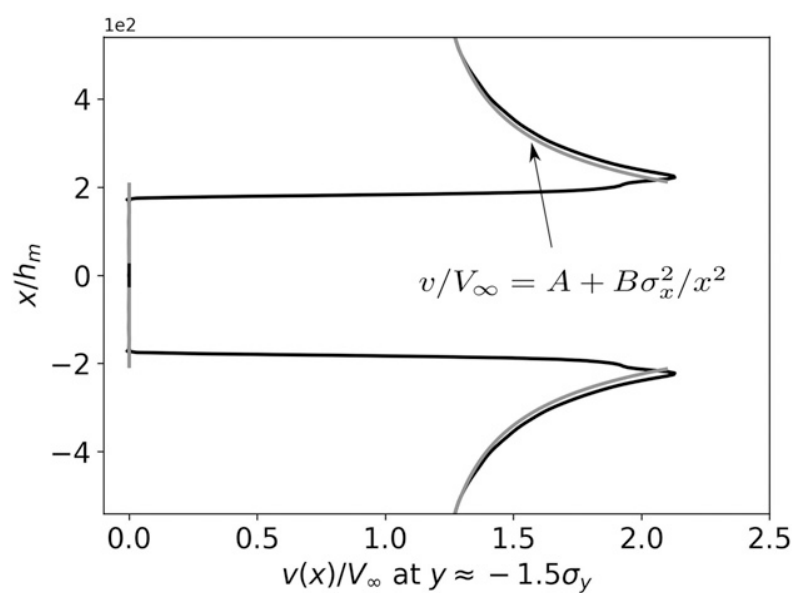

FIG. 11. Streamwise velocity $v(x) / V_{\infty}$ at $t_{\beta}^{\prime}=1.3$ at the vertical level $z=0.1 h_{m}$ and along $y=-1.5 \sigma_{y}$, the half-width of the ridge at $z=0.1 h_{m}$ (indicated as a dashed line in Fig. 10c). Shown in gray is the fit $v / V_{\infty}=A+B \sigma_{x}^{2} / x^{2}$, with $A=1.1$ and $B=1$.

implies that the late-time overflow has a reduced peak speed and correspondingly reduced kinetic energy relative to that at early times. The predicted velocity profile at the new upstream blocking location $y=-y_{b_{f}}$ is shown in Fig. 13 along with the computed profile in the center plane $x=0$ at $t_{\delta}^{\prime}=48.9\left(t_{\beta}^{\prime}=1.3\right)$. The agreement in the peak speed is within $10 \%$ of the newly predicted value. Figure 14 shows the evolution of the transport

$$
Q_{H_{f}}^{\prime}\left(t_{\delta}^{\prime}\right)=\int_{h_{m}-\delta_{f}}^{h_{m}-\delta_{f}+H_{f}} v\left(-y_{b}, z, t_{\delta}^{\prime}\right) d z
$$

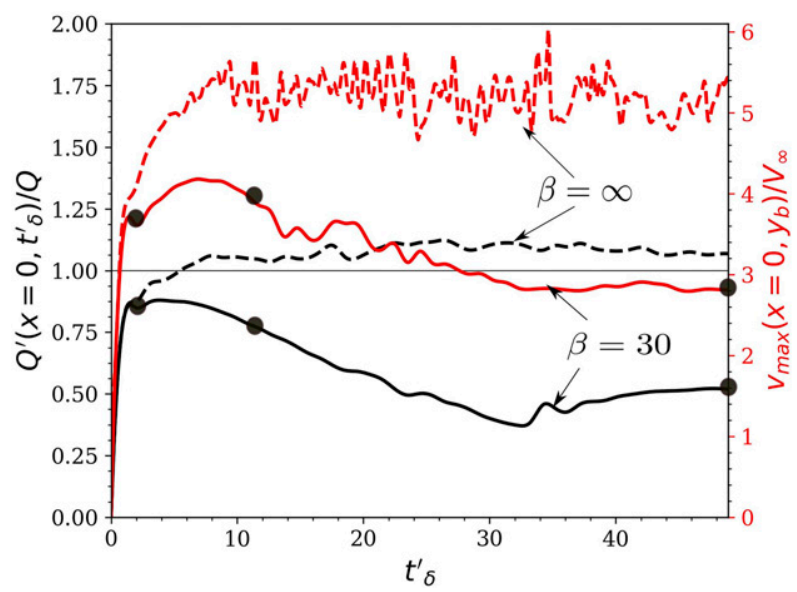

FIG. 12. Upstream volume transport $Q^{\prime}\left(x=0, t_{\delta}^{\prime}\right)$ (black) of the overflow in the center plane of the finite ridge, normalized by the infinite-ridge prediction $Q$; and normalized maximum streamwise velocity $v_{\max }\left(x=0, y_{b}, z, t_{\delta}^{\prime}\right) / V_{\infty}$ (red) at the downstream location $y_{b}$. Dots mark the times $t_{\delta}^{\prime}=2.0,11.7$, and 48.9 for which vertical velocity profiles are shown in Fig. 7.

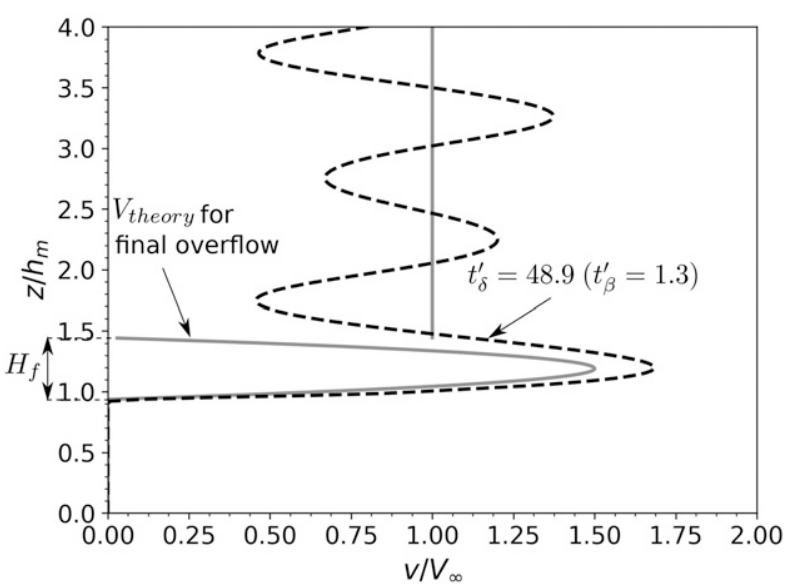

FIG. 13. Vertical profile of the late-time streamwise velocity in the center plane, $x=0$, of the finite ridge at the blocking point, $y=-y_{b_{f}}$, along with the predicted profile based on $H_{f}$.

in a layer of thickness $H_{f}$ above the blocking level $h_{m}-\delta_{f}$ on the center plane, along with the maximum downslope flow speed $v_{\max }\left(y_{b}\right)$. At later times $Q_{H_{f}}^{\prime}$ is within $20 \%$ of $Q_{H_{f}}$ while $v_{\max }\left(y_{b}\right)$ is within $8 \%$ of the Winters and Armi (2014) prediction for $H_{f}$. Thus even at later times, the stratified hydraulic framework describes the properties of the overflow well. We note that the integral in (24) slightly overestimates $Q_{H_{f}}^{\prime}$. This is because in the computed solution (Fig. 13), the overflow merges smoothly with the flow aloft whereas in the theory, there is a discontinuity between these flow components (shown in gray). The essential features of the late-time overflow along the center plane are labeled in Fig. 8c.

Vertical downstream profiles of the streamwise velocity (Fig. 15) offer another view of the evolving downstream flow in the center plane $x=0$ and at $x=0.93 \sigma_{x}$. The flow characteristics are remarkably similar both at and away from the center plane. While the early-time downslope flow closely resembles that observed in the infinite-ridge case (Fig. 5), at later times, the overflow is qualitatively similar, but has reduced transport and kinetic energy. Peak downstream speeds are lower and the locations of the maxima have moved upward to near the crest level $z=h_{m}$.

The combination of a slower overflow and the retention of a cold pool downstream lead to diminished plunging in the lee at $t_{\beta}^{\prime}=\mathscr{O}(1)$ (or equivalently, late inner times $t_{\delta}^{\prime} \gg 1$ ). This is seen in Fig. 16, which shows the time history of the plunging depth, $p_{d}\left(x, t_{\delta}^{\prime}\right)$ in each plane, alongside $p_{d}\left(t_{\delta}^{\prime}\right)$ for the infinite-ridge case. With respect to the inner time scale, the plunging depth overshoots quickly, for example, reaching $2.2 \delta$ in the center plane at $t_{\delta}^{\prime} \approx 2$. However, on the slower splitting 


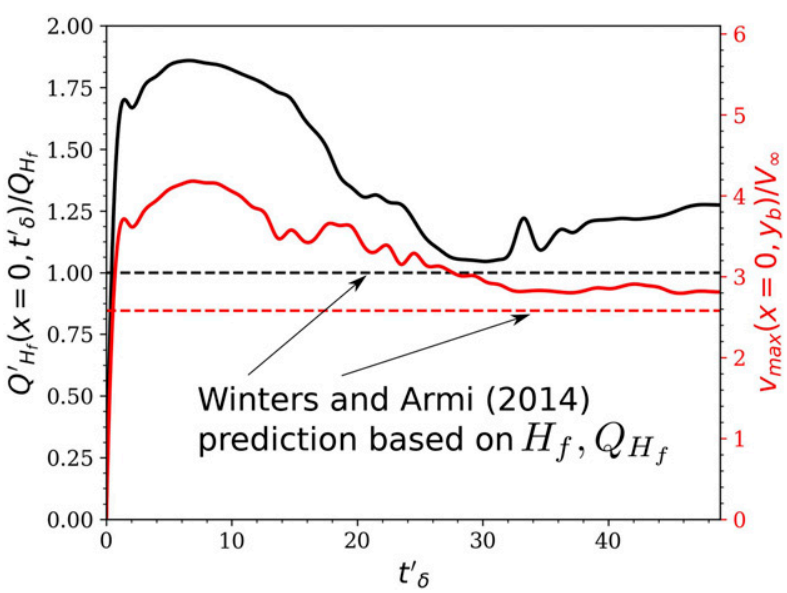

FIG. 14. Upstream volume transport $Q_{H_{f}}^{\prime}\left(t_{\delta}^{\prime}\right)$ in a layer of thickness $H_{f}$ above the blocking level at the center plane of the finite ridge, normalized by the analytical prediction $Q_{H_{f}}$ (black) and normalized maximum streamwise velocity, $v_{\max }\left(y_{b}, z, t_{\delta}^{\prime}\right) / V_{\infty}$ at the downstream location $y_{b}$ (red).

time scale $t_{\beta}$, as the kinetic energy of the overflow decreases (Fig. 15), $p_{d}$ correspondingly levels off to values smaller than $\delta$, both at and away from the center plane.

We now summarize the results of this section as follows: For $0<t_{\delta}^{\prime}<\mathscr{O}(1)$, the development of the flow near the ridge center mimics the $2 \mathrm{D}$ infinite-ridge overflow, both qualitatively and in terms of the quantitative measures of asymmetry $v_{\max }\left(y_{b}\right)$ and $p_{d}$. At intermediate times $\mathscr{O}(1)<t_{\delta}^{\prime}<\mathscr{O}(10)$, the qualitative features of the infinite-ridge solution persist both at and away from the ridge center plane, but the quantitative measures begin to deviate from the infinite-ridge values. In the center plane, $Q^{\prime}$ and $v_{\max }\left(y_{b}\right)$ are still $75 \%$ of the infinite-ridge values for $t_{\delta}^{\prime}$ as high as 12 . On the longer flow-splitting time scale, the energetically weaker overflow is unable to penetrate the cold pool downstream, leading to substantially reduced plunging depths. Nevertheless, acrosscrest asymmetry and downslope flow acceleration persist above the blocking level. The late-time flow is well described as an asymmetric crest-controlled overflow lying above a horizontal splitting flow whose upstream properties follow from potential flow theory.

\section{Discussion}

\section{a. Applicability of the stratified hydraulic framework}

Asymmetry and hydraulic control form the dynamical basis of the infinite-ridge theory of Winters and Armi (2014). In particular, it is a hydrostatic approach that is valid up to arbitrary stretching in the horizontal direction and is applicable for any terrain shape provided the ratio $h_{m} / \sigma_{y}$ is small, as is the case here. A practical (a)

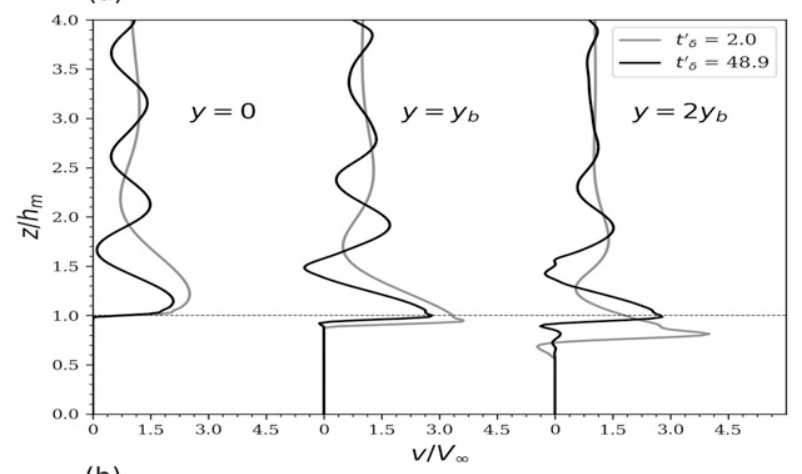

(b)

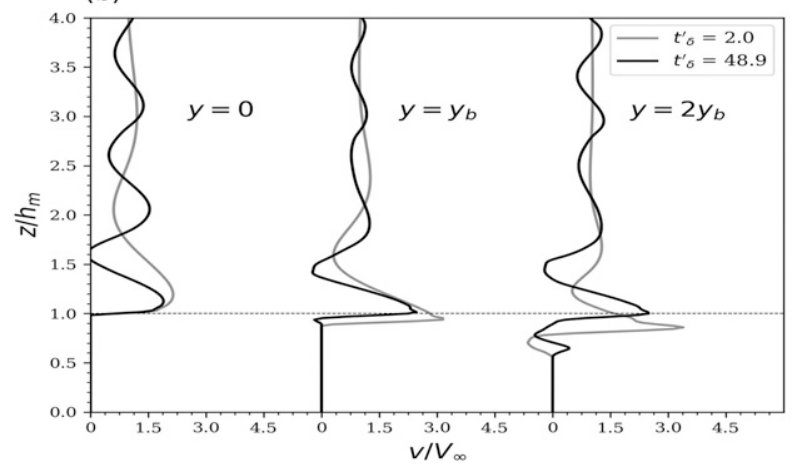

FIG. 15. Vertical profiles of $v\left(x, y, z, t_{\delta}^{\prime}\right)$ at various positions downstream of the upstream blocking location $-y_{b}$ for the finite ridge at two different vertical planes: (a) center plane, $x=0$, and (b) away from the center plane, $x=0.93 \sigma_{x}$. The cross-stream and streamwise positions of the measurement locations are indicated in Figs. 2 and 4a, respectively.

question is, How useful is this hydraulic framework in understanding low Fr flows over long but finite ridges? In this setting, flow over the crest establishes itself on a fast, inner time scale $t_{\delta}$ while flow around the sides develops over a relatively longer time scale $t_{\beta}$.

When the ridge is infinite $(\beta=\infty)$, an asymmetric crestcontrolled flow state is attained quickly, by $t_{\delta}^{\prime}=\mathscr{O}(1)$ (Fig. 3). For a long but finite ridge $(\beta \gg 1)$, the splitting time scale $t_{\beta} \gg t_{\delta}$ and so the development of flow over and around the ridge occur on disparate time scales. As a result, until intermediate times $\mathscr{O}(1)<t_{\delta}^{\prime}<\mathscr{O}(10)$, the flow away from the edges is well approximated as a purely 2D infinite-ridge overflow (Figs. 7 and 12).

By the time $\left[t_{\beta}^{\prime}=\mathscr{Q}(1)\right]$ horizontal splitting effects become significant, the overflow in the ridge interior is already asymmetric and has a parabolic velocity profile upstream of the crest. While its peak speed and thickness begin to decrease with the onset of flow splitting, across-crest asymmetry persists. On the slow splitting time scale $t_{\beta} \gg t_{\delta}$, the overflow retains the essential dynamical features of the infinite-ridge overflow. This flow continues to be optimally controlled and the hydraulic view of the 


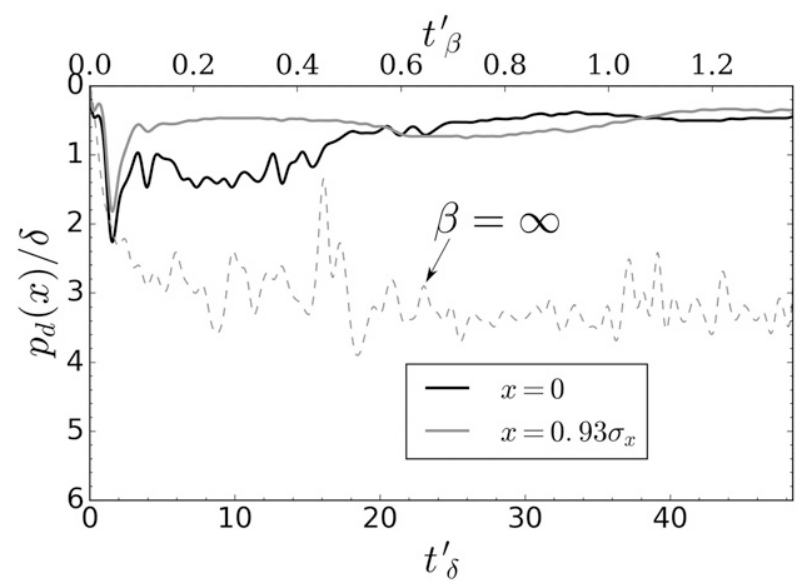

FIG. 16. Plunging depth $p_{d}\left(x, t_{\delta}^{\prime}\right)$ of the lowest streamline that crests the obstacle for the overflow across a finite ridge with $\beta=30$ at $\mathrm{Fr}=0.16$. Also shown is $p_{d}\left(t_{\delta}^{\prime}\right)$ for the corresponding infiniteridge case.

problem remains valid in the neighborhood of the crest. Our findings therefore demonstrate that across-crest asymmetry and hydraulic control, once established, persist even after the low-level flow has split around the sides of the ridge.

The isolating layers in Figs. 4 and 8 form as a result of mixing due to isopycnal overturning aloft, and in the infinite-ridge case, also partly from repeated KelvinHelmholtz overturns at the top of the unstable downslope flow (Jagannathan et al. 2017). However, the hydraulic theory of Winters and Armi (2014) does not provide any insight about the formation of this homogenized isolating layer. Hydraulics also does not explicitly rely on internal mountain wave scales. Rather, the vertical scales of importance, $h_{m}$ and $\delta$, appear only indirectly through the transport equations [e.g., (13)]. While limiting in some ways, the power of this approach is that it fully accounts for nonlinearity, which is not possible in a wave treatment. Further, it produces quantitative predictions for the thickness and peak speed of the overflow, which can be checked against the numerical solutions.

In our experiments, the far-upstream flow speed $V_{\infty}$ and stratification $N$ are constant; that is, these are like impulsively started laboratory towing experiments. This allows for a ready estimate of the modified late-time transport $V_{\infty} H_{f}$ within the overflow of thickness $H_{f}$. However in a geophysical context, these quantities are not usually known a priori and the exact, time-varying upstream flow conditions must be determined using atmospheric soundings. As a more realistic example, one might consider flows with a spinup time $T_{\mathrm{sp}} \gg t_{\delta}$. The Froude number Fr and the inner length scales $\delta$ and $\sigma_{y_{\delta}}=\mathscr{Q}\left(\operatorname{Fr} \sigma_{y}\right)$ will then be slowly evolving functions of time. However note that the inner time scale $t_{\delta}$ built from the instantaneous values of $V_{\infty}$ and $\sigma_{y_{\delta}}$ will remain constant over the spinup period as it is $\mathscr{Q}\left(\sigma_{y} / N h_{m}\right)$ and thus independent of $V_{\infty}$ provided $\mathrm{Fr} \ll 1$. This suggests that when $T_{\mathrm{sp}} \gg t_{\delta}$, the streamwise flow at any time $t<T_{\mathrm{sp}}$ will be hydraulically controlled at the instantaneous Fr, which implies an overflowing layer of slowly expanding thickness and increasing peak speed. Thus while the quantitative details will differ, the essential dynamical character of the flow in the neighborhood of the crest will not change even when the background flow is slowly evolving.

A natural question then, is whether realistic low Fr flows across mountain ridges are characterized by the optimally controlled, parabolically sheared flow profiles predicted by Winters and Armi (2014) and seen in idealized towing experiments. Indeed, such flow features were noted by Armi and Mayr (2007) in their study of continuously stratified flow over the Alps. For example, the sounding at Sterzing (Fig. 16 of Armi and Mayr 2007), taken well after the establishment of deep foehn conditions, reveals a parabolic velocity profile with peak speed of $20 \mathrm{~m} \mathrm{~s}^{-1}$ and thickness of about $3800 \mathrm{~m}$. From the same figure, the blocking depth is seen to be about $500 \mathrm{~m}$ and the mean stratification $N \approx 10^{-2} \mathrm{~s}^{-1}$. Based on the infinite-ridge theory of Winters and Armi (2014), the prediction for the upstream overflow is $H=$ $8 \delta \approx 4000 \mathrm{~m}$ and $v_{\max }=(3 / 2) N H / \pi \approx 19 \mathrm{~m} \mathrm{~s}^{-1}$, which agree well with the observed values.

The stratified hydraulic theory of Winters and Armi (2014), assuming optimal hydraulic control and acrosscrest asymmetry, predicts that the overflow at the blocking location is parabolic in shape. It further relates the thickness of the overflow to the blocking scale, $H=8 \delta$ and predicts its peak speed $v_{\max }=(3 / 2) N H / \pi$ and transport $Q=N H^{2} / \pi$. In simple towing experiments, $H$ is obtained by estimating the overflow transport and equating it to the optimal value $N H^{2} / \pi$. This is trivial for an infinite ridge (see Fig. 1). For long but finite ridges, a straightforward kinematic adjustment to the overflow transport after accounting for flow splitting, yields quantitative predictions for $H$ and $v_{\text {max }}$. We will show in section $6 c$ that by a similar, but algebraically more involved kinematic accounting for the overflow transport, the flow characteristics can be accurately predicted for low Fr flows across composite ridge configurations.

\section{b. Downstream conditions and the cold pool}

A significant point of difference between the finite and infinite-ridge flow solutions is that, in the latter, the flow plunges much deeper into the lee before rebounding back to subcriticality via an internal hydraulic jump (Fig. 4c). Consequently the downslope flow is able to 
accelerate to peak speeds that are more than 5 times the far-upstream flow speed (Fig. 5).

Comparing the isopycnals in Figs. 4 and 8, it is clear that the reason for this difference is the retention, in the case of the finite ridge, of the dense cold pool downstream. This acts as a strong stratification barrier to the plunging overflow, limiting both its speed and penetration depth. By contrast, in the infinite-ridge case, the dense stratified fluid in the lee is swept away after a finite time, and replaced by lighter fluid that overflows the crest. Consequently, the descent of the downslope flow is unimpeded, and it is able to plunge deep in the lee.

This phenomenon is qualitatively similar to the observations of Mayr and Armi (2010) of a foehn event in Owens valley located east of the Sierra Nevada. There they found that diurnal heating, which has the effect of raising the potential temperature of the valley atmosphere, leads to progressively deeper descent of the flow over the course of the day.

In our experiments, the properties of the cold pool are set by the prescribed downstream condition of uniform flow and stratification, and include low-level lee vortices (Fig. 10). While Drazin's (1961) solution fails to predict these lee vortices, Fig. 11 shows that his prediction of layerwise potential flow is nonetheless valid upstream. As in the studies of Smolarkiewicz and Rotunno (1989), Schar and Durran (1997), and Ólafsson and Bougeault (1996), the present simulations were also carried out by imposing free-slip boundary conditions. This suggests that vertical vorticity is produced by a purely inviscid baroclinic mechanism (Smolarkiewicz and Rotunno 1989) at early times. The lee vortices intensify at later times perhaps because of potential vorticity anomalies that develop due to internal dissipation caused by upstream stagnation and flow splitting (Schar and Durran 1997). The role of upstream blocking and flow splitting on orographic wake formation is further discussed by Epifanio and Rotunno (2005). In realistic atmospheric flows, the lee conditions may be affected by other factors such as surface heating or cooling and the presence or absence of secondary topographical features downstream (e.g., Winters 2016).

\section{c. The effect of an abrupt change in ridge elevation}

We now seek to test the applicability of the stratified hydraulic approach in describing the overflow across a composite two-level ridge configuration shown in Fig. 17. This is an infinite ridge with a taller central section for which $\beta=30$ and relative height difference, $\Delta h / h_{1}=1$. Mathematically, the ridge surface is given by (9), where $\sigma_{x}^{*}$ is now the length of the narrow sloping section connecting the two ridge levels and is set to $\sigma_{x} / 120 \ll \sigma_{x}$. The center of the taller section is treated

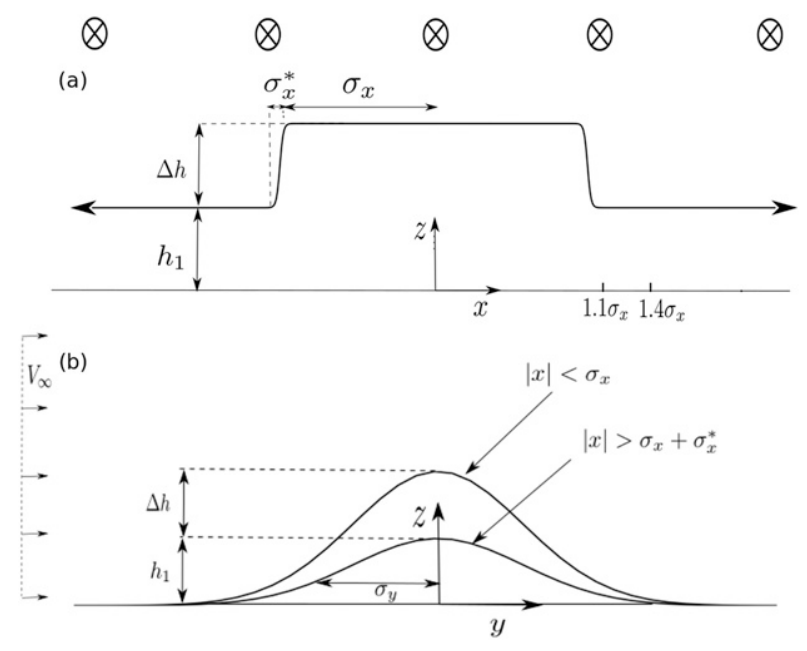

FIG. 17. (a) Front view and (b) side view of an infinite two-level ridge with half-width $\sigma_{y}$, a finite tall central section of height $h_{1}+\Delta h$, and half-length $\sigma_{x}$ with $\beta=\sigma_{x} / \sigma_{y} \gg 1$, straddled on either side by a shorter section of height $h_{1}$ and infinite length. This reduces to the single-level ridge in Fig. 2 when $h_{1}=0$ and $\Delta h=h_{m}$. Figure is not to scale.

as a symmetry boundary and the numerical model configuration and boundary conditions are identical to those in the finite-ridge experiment; $\mathrm{Fr}=V_{\infty} / N h_{1}$ is set to 0.16 , which implies $\mathrm{Fr}=0.08$ for flow approaching the taller section. The details of the numerical experiment are given in Table 2.

\section{1) FAR AWAY FROM THE TALLER SECTION}

The flow well away from the taller section is unaffected by its presence and must hence be identical to that in the infinite-ridge case of section 5a. The fluid beneath the blocking level cannot escape laterally and the low-level transport $V_{\infty}\left(h_{1}-\delta\right)$ augments the overflow transport to match that of a parabolic, optimally controlled flow, as shown in the schematic Fig. 1.

\section{2) Adjacent to the TAlLER SECtion}

At cross-stream distances comparable to $\sigma_{x}$ from the edge of the taller section, the overflow across the shorter section is additionally augmented by the splitting flow around the taller central section. Based on potential flow scaling, (18), for flow around the finite ridge, we hypothesize that, at a vertical level $h_{1}<z^{*}<h_{1}+\Delta h-\delta$, where the ridge half-width is $\sigma_{y_{z} *}$ this splitting flow scales with cross-stream distance as

$v\left(x, y=-\sigma_{y_{z^{*}}}, z^{*}\right) \approx V_{\infty}\left(A+B \frac{\sigma_{x}^{2}}{x^{2}}\right), \quad|x|>\sigma_{x}$.

The experimental domain is the same as in the finiteridge case of section $5 \mathrm{~b}$; therefore $A=1.1$ remains 
(a)

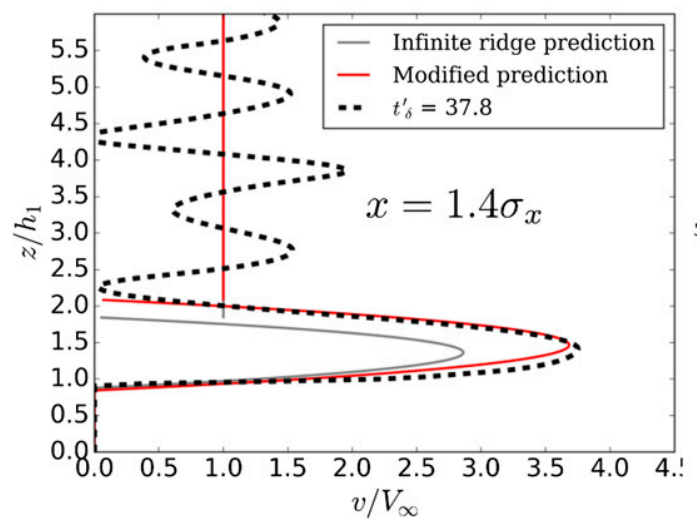

(b)

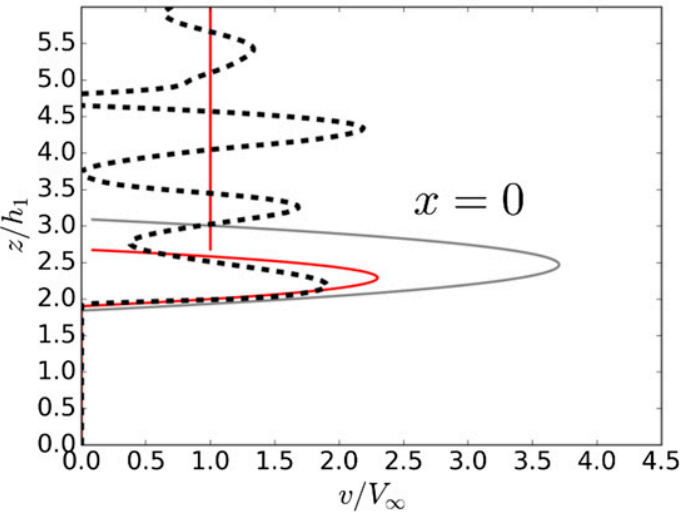

FIG. 18. Computed and predicted vertical profiles of the streamwise velocity at the blocking location, $y=-y_{b}$, of the two-level ridge at (a) the cross-stream location, $x=1.4 \sigma_{x}$ (indicated in Fig. 17), and (b) the center plane, $x=0$, of the taller section. Shown are the naive predictions (gray), obtained by ignoring flow splitting and solving (13) for an infinite ridge, and the modified predictions (red), obtained by solving (26) and (28), which correctly account for flow splitting.

unchanged. However, due to the composite shape of the ridge, we anticipate that the shape factor $B$ will, in general, be different from unity. The splitting flow speed and correspondingly, the overflow transport, decay with increasing cross-stream distance from the edge of the taller section. Assuming optimal crest control and an upstream overflow thickness $H(x)>h_{1}$, the volume conservation equation, (13), for the infinite ridge is modified to

$$
\begin{aligned}
N H^{2}(x) / \pi= & V_{\infty}\left(h_{1}-\delta\right)+V_{\infty}\left(A+B \frac{\sigma_{x}^{2}}{x^{2}}\right)(\Delta h-\delta) \\
& +V_{\infty}(H+\delta-\Delta h)
\end{aligned}
$$

with $\delta=H(x) / 8$. The transport in the overflow is thus enhanced due to flow splitting. Note that as $x \rightarrow \infty$ and correspondingly, $A=1,(26)$ reduces to (13) for the infiniteridge case. We found that, for the case $\Delta h / h_{1}=1$ considered here, the value $B=1.7$ for the shape coefficient produces the most accurate predictions, capturing both the quantitative properties of the overflow and its crossstream decay away from the taller section.

Figure 18a shows a late-time vertical profile of the upstream streamwise velocity at the cross-stream location $x=1.4 \sigma_{x}$ (indicated in Fig. 17a). The gray curve is the naive infinite-ridge prediction based on (13), ignoring ridge geometry and lateral flow splitting, while the red curve is the corrected prediction obtained after modifying the transport calculation according to (26). While the naive prediction underestimates the peak speed and thickness of the overflow by close to $30 \%$, these quantities are predicted almost exactly when flow splitting is accounted for correctly.

\section{3) OVER THE TALLER SECTION}

Since the length of the connecting section is much shorter than the ridge length $\left(\sigma_{x}^{*} \ll \sigma_{x}\right)$, we can, as in section 5a, estimate the excess transport per unit length which is eventually absorbed into the splitting flow. This precisely equals the transport that is lost ahead of the taller section at late times. From (25), on either side of the center plane, this is given by

$Q^{*} \approx \frac{1}{\sigma_{x}} \int_{\sigma_{x}}^{\infty} B V_{\infty} \frac{\sigma_{x}^{2}}{x^{2}}(\Delta h-\delta) d x=B V_{\infty}(\Delta h-\delta)$.

At early times, the blocked transport ahead of the taller section is $V_{\infty}\left(h_{1}+\Delta h-\delta\right)$. Therefore, at later times, the portion of the blocked transport that is accounted for in the overflow aloft is given by $V_{\infty}\left(h_{1}+\Delta h-\delta\right)-$ $B V_{\infty}(\Delta h-\delta)=V_{\infty}\left[h_{1}+\Delta h(1-B)+\delta(B-1)\right]$.

Assuming that this late-time overflow is optimally controlled with upstream thickness $H$, the volume conservation equation for flow over the taller central portion of the ridge is therefore

$$
N H^{2} / \pi=V_{\infty} H+V_{\infty}\left[h_{1}+\Delta h(1-B)+\delta(B-1)\right],
$$

with $\delta=H / 8$ as before. Note that in the limit $\Delta h \rightarrow 0$, there is no splitting flow; the shape factor $B=0$ and we recover the transport equation, (13), for the infinite ridge.

In solving (28), we again use $B=1.7$, the shape factor that produced the best fit for the overflow across the shorter section. Figure $18 \mathrm{~b}$ shows that, consistent with the Winters and Armi (2014) prediction, the computed 
(a)

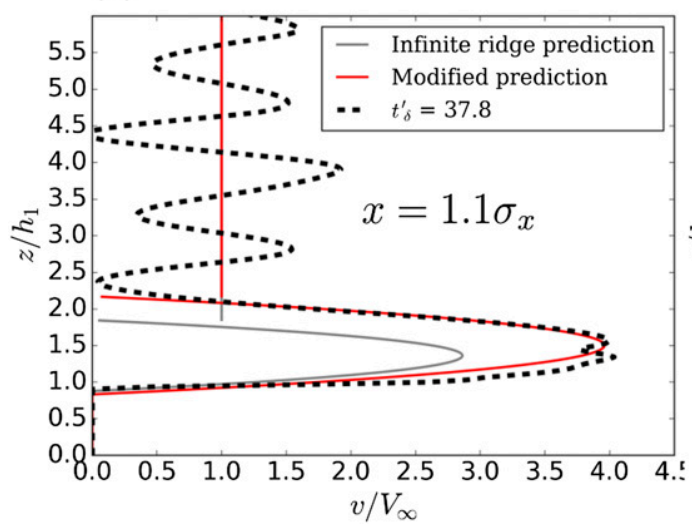

(b)

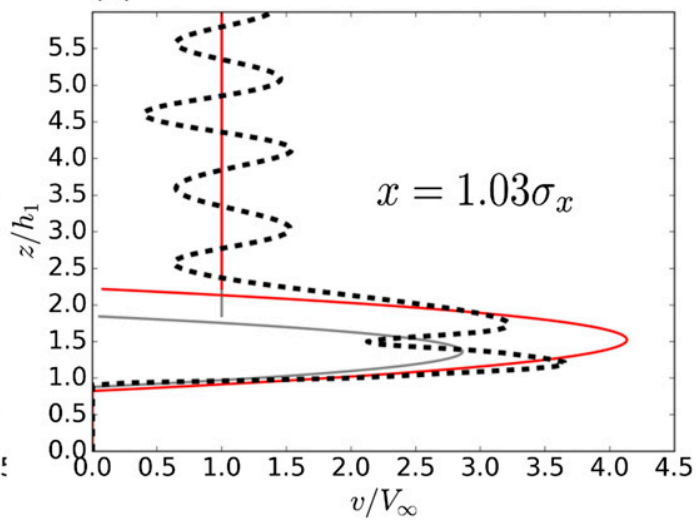

FIG. 19. As in Fig. 18, but at cross-stream locations $y_{1} \leftrightarrow y_{1}^{*}$ nearer to the taller section: (a) $x=1.1 \sigma_{x}$ (indicated in Fig. 17a) and (b) $x=1.03 \sigma_{x}$.

upstream overflow in the center plane, $x=0$, of the taller section has a parabolic profile starting at its blocking level. But whereas the naive prediction substantially overestimates its peak speed and thickness, the modified prediction that accounts for transport lost to the enhanced splitting flow agrees well with the computed flow profile.

\section{4) THE TRANSITION REGION $\sigma_{x}<x \leq 1.1 \sigma_{x}$}

Across the lower portion of the ridge (Fig. 18a), the bottom of the overflow is located at an elevation $z \approx h_{1}-\delta$ while over the taller section $|x|<\sigma_{x}$ it is at $z \approx h_{1}+\Delta h-\delta$ (Fig. 18b). The flow near the abrupt change in ridge height must therefore bridge these two distinct overflows. The upstream velocity profile at the cross-stream location, $x=1.1 \sigma_{x}$, is shown in Fig. 19a. The overflow exhibits two velocity peaks but these are only slightly separated and (26) predicts the bulk properties of the overflow well. Moving closer to the taller section, $x=1.03 \sigma_{x}$, Fig. $19 \mathrm{~b}$ shows that although the total thickness of the overflow is predicted reasonably, its vertical structure is that of two jets with distinct peaks. Thus near the abrupt change in ridge elevation, the overflow is a composite of the short- and tall-section overflows. As a result, even after a kinematic adjustment to the overflow transport, the nearcrest profile cannot be described in terms of a single parabolic overflow as in the infinite-ridge theory of Winters and Armi (2014).

In summary, this two-level ridge example demonstrates that, in the flow regime where upstream influence and blocking are important, the steady overflow upstream of a dynamically tall, long ridge can be obtained by assuming optimal hydraulic control at the crest and coupling it to appropriate kinematic equations for the overflow transport. The details of the ridge geometry matter only to the extent that they modify the transport calculations. While the plunging depth of the overflow and its eventual separation in the lee are influenced by downstream conditions, a layerwise solution of Bernoulli's equation, as in Winters and Armi (2014) furnishes a complete description of the asymmetric overflow in the neighborhood of the crest.

\section{Limitations and extensions}

\section{a. Coriolis effects}

As shown in the simulations, the hydraulic flow component develops on the short time scale $t_{\delta}$ while the lowlevel splitting dynamics is established over a longer time scale $t_{\beta} \gg t_{\delta}$. Thus rotational effects play no role provided that $t_{\beta} \ll \mathscr{O}(1 / f)$, where $f$ is the Coriolis frequency. In many midlatitude atmospheric flows characterized by low Fr and large $\beta$, for example, with $N=10^{-2} \mathrm{~s}^{-1}$ and $V_{\infty}=10 \mathrm{~m} \mathrm{~s}^{-1}$ and mountain dimensions $h_{m}=4 \mathrm{~km}, \sigma_{y}=$ $20 \mathrm{~km}$, and $\sigma_{x}=200 \mathrm{~km}$ (yielding $\mathrm{Fr}=0.25$ and $\beta=10$ ), the corresponding $t_{\delta}$ can be shown to be well under an hour while $t_{\beta}$ is about a quarter of an inertial period. Thus both the crest-controlled overflow and lateral splitting flow are established before Coriolis effects become significant.

The layerwise upstream potential flow solution has also been realized in laboratory towing experiments (e.g., Brighton 1978; Hunt and Snyder 1980). Yet there are other geophysical situations where the flow-splitting time scale may be comparable to or larger than an inertial period. When the evolution of the low-level splitting flow is constrained by rotation, its horizontal scale is no longer set by the mountain half-length $\sigma_{x}$. Rather, as hypothesized by Pierrehumbert and Wyman (1985) and confirmed by Wells et al. (2005), geostrophic imbalance leads 


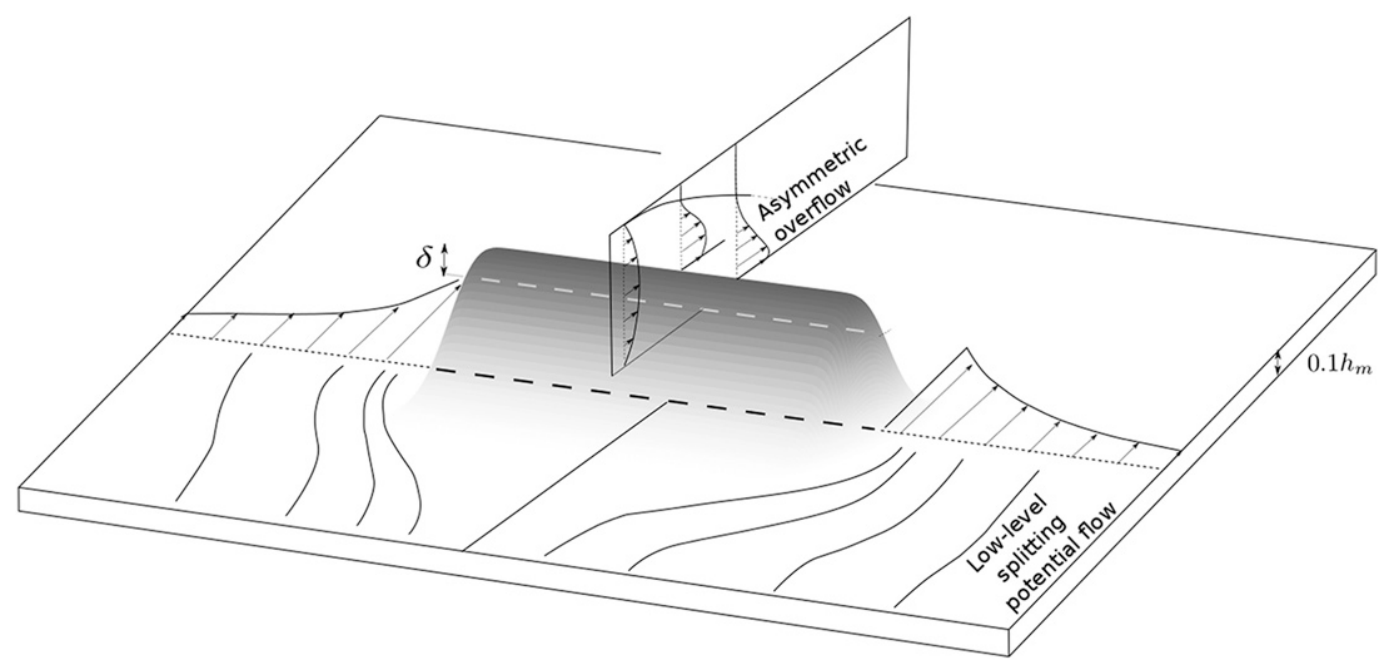

FIG. 20. Schematic depicting the essential features of low Froude number over and around a long mountain ridge of height $h_{m}$. The flow has two distinct components. Above the blocking level is an overflow that is asymmetric and hydraulically controlled at the crest. Below the blocking scale $\delta$ from the crest is a layerwise horizontal splitting flow that is potential-like upstream. The downstream penetration of the overflow is dependent on lee conditions and in our simple towing experiments, is limited due to the retention of a dense cold pool.

to the development of a mountain-parallel barrier jet trapped within a deformation radius $N h_{m} / f$ of the mountain. This disrupts the symmetry of the upstream splitting flow as well as the lee vortices, as can also be seen in the simulations of Wells et al. (2005). Moreover, Ólafsson and Bougeault (1997) note that rotational asymmetry can affect the overall mountain drag relative to nonrotating flows. Nevertheless, while the direct applicability of our framework to rotationally constrained atmospheric flows may be limited, our results show that hydraulically controlled overflows are established over relatively short time scales and that the splitting effect for finite ridges manifests itself as a reduction in the transport of the overflowing layer.

\section{b. Simple turbulence model}

The focus of this study is on the dynamics of upstream blocking, hydraulic crest control, and low-level flow splitting. These processes are laminar and therefore insensitive to the presence or absence of any turbulence closure model. Downstream of the crest, the supercritical flow is unstable to overturning shear instabilities. We resolve the formation of these instabilities but model the subsequent turbulence using a simple closure scheme that removes gridscale variability. The geometrical details of the overturns and the isolating layer possibly depend on the choice of turbulent parameterization. Nevertheless the good quantitative agreement with the predictions of stratified hydraulic theory shows that, regardless of the turbulence model, it is a robust dynamical framework for analyzing and interpreting the flow solutions. Winters
(2016) employed a closure scheme similar to the one used here in a higher-resolution LES treatment of these processes in topographically controlled flows over an infinite ridge. A comparable treatment for the finite-ridge case in which a hydraulically controlled overflow occurs in conjunction with lateral flow splitting is beyond our current computing capability.

\section{c. The nature of the flow aloft}

In both the infinite and finite-ridge cases, we noted that the flow above the controlled overflowing layer is characterized by spatial oscillations with vertical wavelength approximately $2 \pi V_{\infty} / N$ (appendix B). While the hydraulic theory of Winters and Armi (2014) assumes that the controlled overflowing layer is decoupled from the overlying flow, it does not say anything about the possibility of wave excitation aloft as a response to the plunging overflow. Therefore the present analysis does not yield any insight about the amplitude of this wavelike flow nor its dynamical connection to the controlled overflow beneath.

One interpretation of the layered structure aloft (e.g., Fig. 4c) is as follows. The flow in the layer immediately above the controlled overflow responds to the "virtual topography" formed by the plunging overflow and is also asymmetric across the crest. The layers further aloft respond in a similar fashion. A similar response to virtual topography was noted by Armi and Mayr (2015) in their observations in the Sierras of hydraulically controlled flows with a descending temperature inversion at the top of the overflowing layer. 


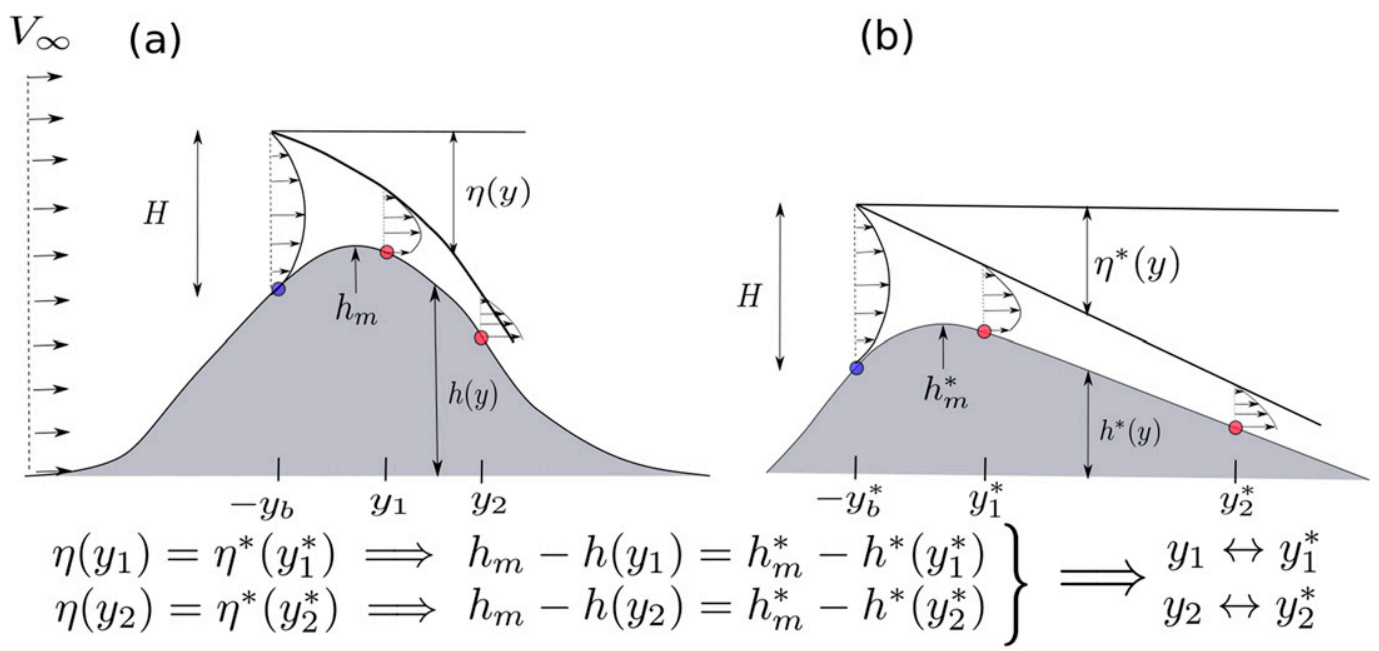

FIG. A1. Schematic illustrating how blocked controlled flows over an arbitrary topography are mapped to the Winters and Armi (2014) solution. (a) Low Fr flow of specified upstream velocity $V_{\infty}$ and uniform stratification $N$ incident on an arbitrary topography $h(y)$ produces a hydraulically controlled overflow with a bifurcating streamline $\eta(y)$ of unknown slope as indicated. The blocking location is at $y=-y_{b}$ and the upstream overflow depth $H$ is determined by solving the transport equation [e.g., (13)]. The resulting streamwise velocity profiles at arbitrary downstream locations $y=y_{1}$ and $y_{2}$ are also shown. (b) Schematic of the Winters and Armi (2014) solution. The upstream overflow thickness $H$ found in (a) is now specified and a bifurcating streamline of constant slope is imposed. Downstream of the blocking location $y=-y_{b}^{*}$, layerwise integration of Bernoulli's equation yields the flow solution within the controlled layer and also produces a corresponding terrain shape $h^{*}(y)$ with a single peak. On either side of the crest, the height by which the streamline has dropped $\eta^{*}(y)$ and the height of the terrain surface relative to the blocking point (blue dot) is a unique one-to-one map. Thus, the solution obtained at any location, for example, $y=y_{1}^{*}$, can be mapped to that at $y=y_{1}$ in (a), that is, $y_{1} \leftrightarrow y_{1}^{*}$ provided $h\left(y_{1}\right)-h\left(-y_{b}\right)=h^{*}\left(-y_{b}^{*}\right)-h^{*}\left(y_{1}^{*}\right)$.

\section{d. Extensions to other small Fr and large $\beta$ flows}

In the infinite-ridge case, lowering Fr further while keeping $h_{m}$ fixed shrinks the blocking scale $\delta$ and hence, also $\sigma_{y_{\delta}}$ and $t_{\delta}$. This leads to quicker establishment of hydraulic control with a correspondingly thinner overflow. Increasing Fr has the exact opposite effect.

For a finite ridge, the $(\mathrm{Fr}, \beta)$-regime diagram (e.g., Smith 1989; Lin 2007) indicates that the flow behavior at low Fr and large $\beta$ is uncertain. That is, the precise parameter space over which both flow splitting and "wave breaking" occur is unknown. The present study suggests another interpretation of this regime diagram. For a finite ridge, changing $\mathrm{Fr}$ and $\beta$ essentially has the effect of making $t_{\delta}$ and $t_{\beta}$ more or less disparate relative to one another. We have seen that blocking and hydraulic control are established on the short time scale $t_{\delta}$. Assuming that wave breaking triggers a transition to the controlled state (e.g., Baines 1998), we may conjecture that flow splitting and lee vortices are accompanied by a crest-controlled overflow when $t_{\beta}$ is about an order of magnitude larger than $t_{\delta}$. When these two time scales become comparable, the overflow will be subcritical and the flow then falls in the flow-splittingonly regime.

\section{Concluding remarks}

Across-crest asymmetry and hydraulic control are persistent features of low Fr flows past long ridges. On a short time scale, upstream blocking imparts a fundamentally nonlinear and asymmetric character to the flow over the crest. This asymmetry persists even on the longer time scale over which the low-level splitting flow is established. The flow is therefore composed of two distinct dynamical components as depicted in Fig. 20. The assumption of optimal crest control along with the recognition that the upstream splitting flow is potential-like further allows for an accurate reformulation of the nearcrest overflow.

Acknowledgments. This work was supported by the National Science Foundation through Grant AGS-1540585. We would like to acknowledge high-performance computing support from Yellowstone (ark:/85065/d7wd3xhc) provided by NCAR's Computational and Information Systems Laboratory, sponsored by the National Science Foundation. XSEDE computing resources were made available under Grant TG-OCE120004. We thank Dr. Richard Rotunno for his insightful feedback on an early version of the manuscript. The comments of an 

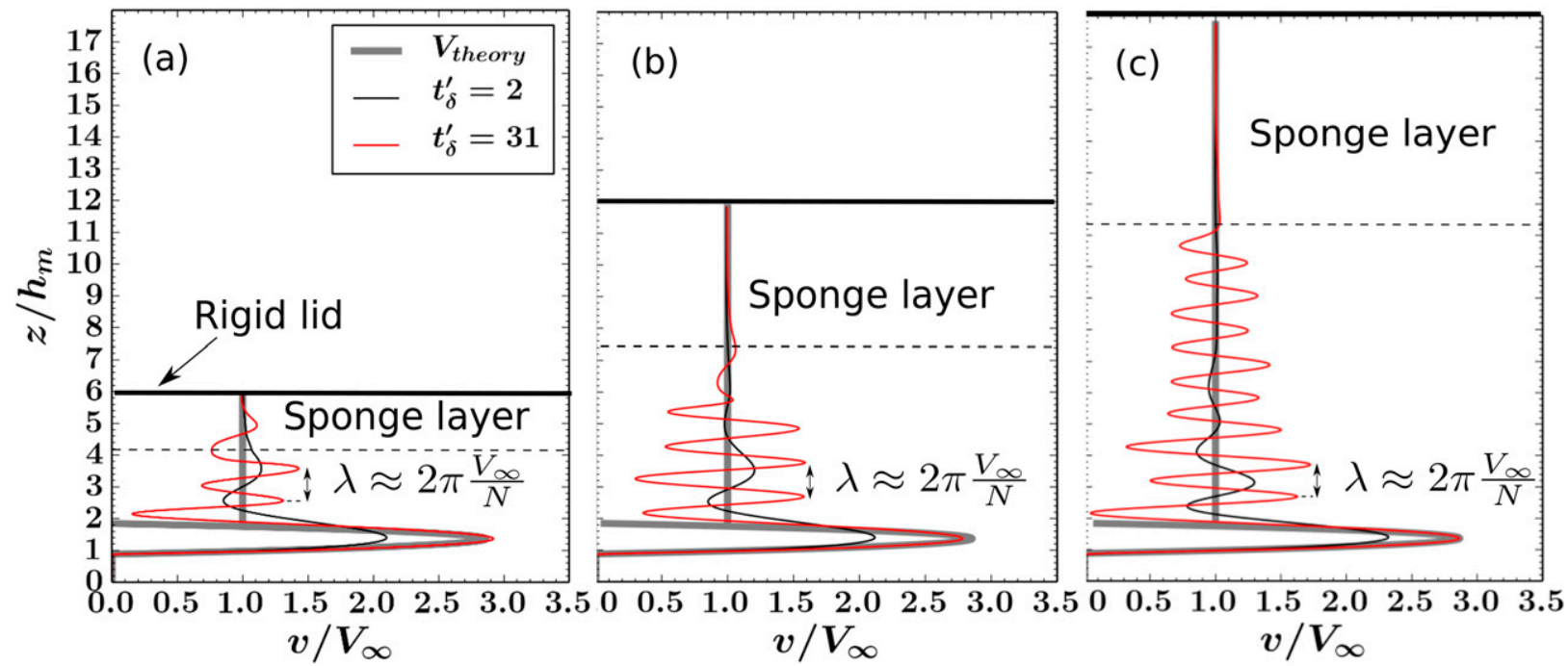

FIG. B1. Vertical profiles of the streamwise velocity at the blocking point $y \approx-y_{b}$ along with the analytical prediction of Winters and Armi (2014) (gray) for $\mathrm{Fr}=0.16$ flow over an infinite ridge with domain height and Rayleigh damping layer thickness respectively set to (a) $6 h_{m}$ and $1.8 h_{m}$, (b) $12 h_{m}$ and $4.5 h_{m}$, and (c) $18 h_{m}$ and $6.7 h_{m}$.

anonymous referee motivated the addition of section 3 and appendix A.

\section{APPENDIX A}

\section{Mapping the Winters and Armi (2014) Solution to Arbitrary Terrain Shapes}

In the numerical flow solutions we present, the specified pieces of information are the topography $h(y)$ and upstream flow conditions $V_{\infty}$ and $N$. From these, we estimate the overflow transport $Q$ and set up a quadratic equation for the upstream overflow thickness $H$ as in (13). After determining $H$, it is specified as an input to the Winters and Armi (2014) model along with $N$ and some small, constant slope for the bifurcating streamline. The model then produces flow solutions downstream of the blocking point, including an implied terrain shape $h^{*}(y)$ with peak height $h_{m}^{*}$. On either side of the crest, the solution additionally generates a unique one-to-one map between the drop of the bifurcating streamline $\eta^{*}(y)$ and the height of any point along the terrain surface relative to the blocking level $h^{*}(y)-\left(h_{m}^{*}-H / 8\right)$. This is depicted schematically in Fig. A1. The flow solutions at arbitrary downstream locations, for example, $y=y_{1}$ and $y_{2}$ along the given topography $h(y)$ in Fig. A1a, can be mapped to $y=y_{1}^{*}$ and $y_{2}^{*}$, respectively, along the implied terrain shape produced by the Winters and Armi (2014) model, shown in Fig. A1b. Thus the analytical flow solutions of Winters and Armi (2014) are valid for any arbitrary hydrostatic topography.

\section{APPENDIX B}

\section{Sensitivity of the Flow Structure Aloft to Domain Height and Sponge-Layer Thickness ${ }^{1}$}

The question of whether the wavelike flow above the controlled overflow is caused by wave trapping as a result of imperfect radiation at the upper boundary and its possible effects on the overflow were investigated further. If the upper boundary in effect behaves as a rigid lid, then only certain quantized modes can exist and the quantization will dependent on the height of the domain.

To test whether the quantitative properties of the controlled overflowing layer and the oscillatory flow aloft (e.g., in Fig. 3a) depend sensitively on the choice of the domain height and sponge-layer thickness, we repeated the $\mathrm{Fr}=0.16$ infinite-ridge simulation for three different configurations of domain and spongelayer depths. The sponge layer is designed to absorb the most energetic upward-propagating waves of vertical scale about $h_{m}$. In the original case, the vertical height of the domain $L_{z}=6 h_{m}$ and the sponge layer has a thickness $1.8 h_{m}$. In the other two cases, we set $L_{z}=12 h_{m}$ and $18 h_{m}$, with much deeper damping layers, of thickness $4.5 h_{m}$ and $6.7 h_{m}$, respectively. The vertical profile of the streamwise velocity at the blocking point (Fig. B1) shows that the quantitative properties of the

\footnotetext{
${ }^{1}$ Motivated by Dr. Richard Rotunno's insightful comment on an early version of the manuscript.
} 
controlled overflow are unchanged and the oscillatory flow structure aloft also persists in all cases. Further, Fig. B1 shows that the vertical wavelength of this wavelike flow is independent of domain height and is around $\lambda \approx 2 \pi V_{\infty} / N$ in all cases.

\section{REFERENCES}

Armi, L., and G. J. Mayr, 2007: Continuously stratified flows across an Alpine crest with a pass: Shallow and deep föhn. Quart. J. Roy. Meteor. Soc., 133, 459-477, https://doi.org/ 10.1002/qj.22.

_ and - 2015: Virtual and real topography for flows across mountain ranges. J. Appl. Meteor. Climatol., 54, 723-731, https://doi.org/10.1175/JAMC-D-14-0231.1.

Baines, P. G., 1977: Upstream influence and Long's model in stratified flows. J. Fluid Mech., 82, 147-159, https://doi.org/10.1017/ S0022112077000573.

__ 1987: Upstream blocking and airflow over mountains. Annu. Rev. Fluid Mech., 19, 75-95, https://doi.org/10.1146/ annurev.fl.19.010187.000451.

_ 1998: Topographic Effects in Stratified Flows. Cambridge University Press, 500 pp.

_ and K. P. Hoinka, 1985: Stratified flow over two-dimensional topography in fluid of infinite depth: A laboratory simulation. J. Atmos. Sci., 42, 1614-1630, https://doi.org/10.1175/15200469(1985)042<1614:SFOTDT>2.0.CO;2.

Bauer, M. H., G. J. Mayr, I. Vergeiner, and H. Pichler, 2000: Strongly nonlinear flow over and around a three-dimensional mountain as a function of the horizontal aspect ratio. J. Atmos. Sci., 57, 3971-3991, https://doi.org/10.1175/1520-0469(2001) $058<3971$ :SNFOAA $>2.0 . \mathrm{CO} ; 2$.

Belušić, D., M. Pasarić, and M. Orlić, 2004: Quasi-periodic bora gusts related to the structure of the troposphere. Quart. J. Roy. Meteor. Soc., 130,1103-1121, https://doi.org/10.1256/ qj.03.53.

Brighton, P., 1978: Strongly stratified flow past three-dimensional obstacles. Quart. J. Roy. Meteor. Soc., 104, 289-307, https:// doi.org/10.1002/qj.49710444005.

Chow, F. K., S. F. De Wekker, and B. J. Snyder, 2012: Mountain Weather Research and Forecasting: Recent Progress and Current Challenges. Springer Science and Business Media, 750 pp., https://doi.org/10.1007/978-94-007-4098-3.

Drazin, P. G., 1961: On the steady flow of a fluid of variable density past an obstacle. Tellus, 13, 239-251, https://doi.org/10.3402/ tellusa.v13i2.9451.

Durran, D. R., 1990: Mountain waves and downslope winds. Atmospheric Processes over Complex Terrain, Meteor. Monogr. No. 45, Amer. Meteor. Soc., 59-81.

Epifanio, C. C., and D. R. Durran, 2001: Three-dimensional effects in high-drag-state flows over long ridges. J. Atmos. Sci., $\mathbf{5 8}$ 1051-1065, https://doi.org/10.1175/1520-0469(2001)058<1051: TDEIHD $>2.0 . \mathrm{CO} ; 2$.

- , and R. Rotunno, 2005: The dynamics of orographic wake formation in flows with upstream blocking. J. Atmos. Sci., 62 , 3127-3150, https://doi.org/10.1175/JAS3523.1.

Hanazaki, H., 1988: A numerical study of three-dimensional stratified flow past a sphere. J. Fluid Mech., 192, 393-419, https://doi.org/ 10.1017/S0022112088001910.

Hunt, J., and W. Snyder, 1980: Experiments on stably and neutrally stratified flow over a model three-dimensional hill. J. Fluid Mech., 96, 671-704, https://doi.org/10.1017/S0022112080002303.
Jackson, P. L., G. Mayr, and S. Vosper, 2013: Dynamically-driven winds. Mountain Weather Research and Forecasting, F. K. Chow, S. F. De Wekker, and B. J. Snyder, Eds., Springer, 121-218, https://doi.org/10.1007/978-94-007-4098-3_3.

Jagannathan, A., K. B. Winters, and L. Armi, 2017: Stability of stratified downslope flows with an overlying stagnant isolating layer. J. Fluid Mech., 810, 392-411, https://doi.org/10.1017/ jfm.2016.683.

Lin, Y.-L., 2007: Mesoscale Dynamics. Cambridge University Press, 630 pp., https://doi.org/10.1017/CBO9780511619649.

Mayr, G. J., and L. Armi, 2010: The influence of downstream diurnal heating on the descent of flow across the Sierras. J. Appl. Meteor. Climatol., 49, 1906-1912, https://doi.org/ 10.1175/2010JAMC2516.1.

Ólafsson, H., and P. Bougeault, 1996: Nonlinear flow past an elliptic mountain ridge. J. Atmos. Sci., 53, 2465-2489, https://doi.org/ 10.1175/1520-0469(1996)053<2465:NFPAEM > 2.0.CO;2.

_, and _ 1997: The effect of rotation and surface friction on orographic drag. J. Atmos. Sci., 54, 193-210, https://doi.org/ 10.1175/1520-0469(1997)054<0193:TEORAS > 2.0.CO;2.

Peltier, W., and J. Scinocca, 1990: The origin of severe downslope windstorm pulsations. J. Atmos. Sci., 47, 2853-2870, https://doi.org/10.1175/1520-0469(1990)047<2853:TOOSDW> 2.0. $\mathrm{CO} ; 2$

Pierrehumbert, R., and B. Wyman, 1985: Upstream effects of mesoscale mountains. J. Atmos. Sci., 42, 977-1003, https://doi.org/ 10.1175/1520-0469(1985)042<0977:UEOMM > 2.0.CO;2.

Queney, P., 1948: The problem of air flow over mountains: A summary of theoretical studies. Bull. Amer. Meteor. Soc., 29, 16-26, https://doi.org/10.1175/1520-0477-29.1.16.

Schar, C., and D. R. Durran, 1997: Vortex formation and vortex shedding in continuously stratified flows past isolated topography. J. Atmos. Sci., 54, 534-554, https://doi.org/10.1175/15200469(1997)054<0534:VFAVSI $>2.0$. CO;2.

Sheppard, P., 1956: Airflow over mountains. Quart. J. Roy. Meteor. Soc., 82, 528-529, https://doi.org/10.1002/qj.49708235418.

Smith, R. B., 1980: Linear theory of stratified hydrostatic flow past an isolated mountain. Tellus, 32, 348-364, https://doi.org/ 10.3402/tellusa.v32i4.10590.

_ 1985: On severe downslope winds. J. Atmos. Sci., 42, 25972603, https://doi.org/10.1175/1520-0469(1985)042<2597:OSDW> 2.0.CO;2.

1989: Hydrostatic airflow over mountains. Advances in Geophysics, Vol. 31, Academic Press, 1-41, https://doi.org/10.1016/ S0065-2687(08)60052-7.

Smolarkiewicz, P. K., and R. Rotunno, 1989: Low Froude number flow past three-dimensional obstacles. Part I: Baroclinically generated lee vortices. J. Atmos. Sci., 46, 1154-1164, https://doi.org/10.1175/1520-0469(1989)046<1154:LFNFPT> 2.0.CO;2.

Wells, H., S. Webster, and A. Brown, 2005: The effect of rotation on the pressure drag force produced by flow around long mountain ridges. Quart. J. Roy. Meteor. Soc., 131, 1321-1338, https://doi.org/10.1256/qj.04.37.

Winters, K. B., 2016: The turbulent transition of a supercritical downslope flow: Sensitivity to downstream conditions. J. Fluid Mech., 792, 997-1012, https://doi.org/10.1017/jfm.2016.113. , and A. de la Fuente, 2012: Modelling rotating stratified flows at laboratory-scale using spectrally-based DNS. Ocean Modell., 49-50, 47-59, https://doi.org/10.1016/j.ocemod.2012.04.001. , and L. Armi, 2014: Topographic control of stratified flows: Upstream jets, blocking and isolating layers. J. Fluid Mech., 753, 80-103, https://doi.org/10.1017/jfm.2014.363. 